\title{
Casing-groove Optimisation for Stall Margin in a Transonic Compressor Rotor
}

\begin{abstract}
Purpose - The aims of this paper are two-fold. The first goal is to understand aerodynamic blockage related to the near casing flow in a transonic axial compressor using numerical simulations. Secondly, the design of an optimum casing groove for stall margin improvement is attempted using a surrogate-optimisation technique.
\end{abstract}

Design/methodology/approach - A blockage parameter $(\Psi)$ is introduced to quantify blockage across the blade domain. A surrogate-optimisation technique is then employed to find the optimum casing groove design that minimises blockage at an axial location where blockage is maximum at near stall conditions.

Findings -An optimised casing groove that improves the stall margin by about $1 \%$ can be found through optimisation of the blockage parameter $(\Psi)$.

Originality/value - Optimising for stall margin is rather lengthy and computationally expensive as the stall margin of a compressor will only be known once a complete compressor map is constructed. It is shown here that the cost of the optimisation can be reduced by using a suitably defined blockage parameter as the optimising parameter.

Keywords Axial Compressor, Stall Margin, Optimisation

Paper type Research paper

\section{Introduction}

When the operating mass flow of the compressor is progressively reduced below a certain critical value the compressor becomes subjected to instabilities known as rotating stall. This is characterised by a sudden loss of useful pressure rise through the compressor.

Enhancing the stability limit of the compressor using passive wall treatments has been a subject of interest for many years. Hathaway (2007) provided a compilation of past attempts in using passive casing treatments to improve the stability of compressors. The potential use of circumferential casing grooves for improving the stall margin has also been demonstrated in the past (Houghton and Day, 2010; Sakuma et al., 2013; Shabbir and Adamczyk, 2004) in both low and high-speed compressors. Shabbir et al. (2004) explained that unlike for a smooth (untreated) casing, casing grooves provide a path for the blocked flow to be transported radially which helps to balance the axial pressure force and hence delay the onset of stall. In a lowspeed compressor, Houghton et al. (2010) showed that the positioning of the casing groove affects the stall margin improvement and compressor efficiency. It was shown that the casing 
groove gives substantial stall margin improvement (SMI) with minor efficiency penalty when it is located close to the tip leading edge (LE) or near mid-chord. For a high-speed compressor, Sakuma et al. (2013) found that the proper sizing and positioning of the groove may further improve the stall margin. It was shown that deeper casing groove performs the best when positioned at 20\% $c_{a x, t}$. The discrepancy between (Houghton and Day, 2010) and (Sakuma et al., 2013) in terms of the best casing groove positioning, can be linked to the difference in the build-up of low momentum fluid or blockage near the tip region as the compressor approaches the stability limit. Past studies from low and high-speed compressors have established the link between the growth of blockage due to tip leakage vortex breakdown (Furukawa et al., 1999; Yamada et al., 2007) and radial vortex (Brandstetter and Schiffer, 2018; Inoue et al., 1999) to the onset of stall. The presence of a radial vortex has been shown both numerically (Pullan et al., 2015) and experimentally (Inoue et al., 1999) within a low-speed compressor environment. However to the author's best knowledge the existence of a radial vortex within a high-speed compressor has only been shown experimentally by Brandstetter and Schiffer (2018) so far.

Unlike high-speed compressors, low-speed compressors are devoid of compressibility effects such as shock-tip leakage vortex (TLV) interaction. In high-speed compressors, passage shock-TLV interaction as reported by Suder and Celestina (1996) is found to be responsible for the accumulation of blockage at the tip region which is about two to three times higher than the blockage in the core flow region. It was also reported that the passage shock-TLV interaction results in a blockage region that led to high incidence angle at the tip region. This would then suggest a possible relationship between the blockage and the selection of the best casing groove position. In other words, the ability of the casing groove to enhance the stability limit of a compressor may be related to flow in the tip region where blockage is maximum. Although, as previously mentioned, Houghton and Day (2010) and Sakuma et al. (2013) found their casing grooves to perform best at certain axial locations, they did not explicitly link their best case scenario to the position of the blockage in these studies.

In the numerical study presented in this paper, a particular interest is given to understanding the blockage caused by passage shock-TLV interaction that is common in highspeed compressors. The first aim of this paper is to understand the near casing aerodynamics and its relation to blockage. For this purpose, a blockage quantification method will be introduced in order to map the blockage distribution. It is known from Houghton and Day (2010) and Sakuma et al. (2013) that there is a trade-off between SMI and compressor efficiency penalty due to casing grooves. Thus, the second aim of this study is to find the 
optimum groove design that balances SMI and the efficiency penalty. A surrogate-based optimisation method will be employed to determine the ideal size and position of a single circumferential casing groove.

In design optimisation problems, the role of the optimiser algorithm is to search for the optimum design by evaluating the objective function $(f)$. Since there would be hundreds, if not thousands of design possibilities, obtaining the output of an objective function from high fidelity simulations is not feasible due the associated computational cost. Surrogate modelling, as used in the present study, provides an alternative way of obtaining the desired output by constructing a robust approximation of the objective function with less computing resources. This technique has been used in aerodynamic design optimisation problems such as those reported by Pakatchian et al. (2019) and Simpson et al. (2001).

Finally, it is not always clear what parameters need to be optimised for achieving SMI from single test cases of geometry variation. For a given test-case geometry, stall margin can only be established after constructing a complete compressor map. This makes the optimisation process rather lengthy if SMI is chosen as the optimisation parameter as one has to conduct several numerical simulations to construct the complete compressor map for each of the geometry considered. The paper, therefore, explores the use of a blockage parameter $(\Psi)$, the reduction of which is used as one of the objectives of the optimisation process such that the resulting geometry leads to SMI. The full compressor map is only constructed for the resulting optimised geometry in order to evaluate the SMI achieved. Through this approach, the computation cost of the optimisation is expected to be reduced.

Figure 1 Meridional cut-view of NASA Rotor 37

\section{Computational Method}

The numerical domain is based on an isolated transonic axial compressor rotor, NASA Rotor 37. Rotor 37 is part of the inlet stage of an advanced core compressor with 20:1 pressure ratio (Suder, 1996). Figure 1 shows the meridional view of a single passage numerical domain used for this study. A single passage simulation is appropriate since this numerical study is only performed at conditions within the stability limit where periodic flow conditions are valid. Aerodynamic properties and specifications of this rotor blade are obtained from a CFD blind test case published by Denton (1997) and are summarised in Table 1.

Table 1 Design parameters of Rotor 37 
Figure 2 Grid points generated in the tip region

\section{Geometry and Grid}

The computational grid consists of about 4 million nodes using a combination of $\mathrm{H}$ and O-grids. The grid resolution is chosen after performing a grid sensitivity analysis using 2, 4 and 6 million grid points. Changes to the calculated flow solution is found to be relatively small hence the domain with 4 million grid points is chosen for this study. The grid resolution chosen is also benchmarked against other steady RANS numerical studies in the literature using Rotor 37. For comparison, the number of grid points used by Hah and Loellbach (1999) and Sakuma et al. (2013) was 0.5 million and 1.5 million grid points, respectively. The H-grid that covers the entire computation domain has $248 \times 86 \times 148$ grid points in the axial, pitch and radial direction, respectively. As for the rotor blade, there are 125 axial grid points along the blade pressure/suction surface and 115 radial grid points from hub to tip. The tip gap as shown in Figure 2 has 30 radial grid points to ensure that the flow features inside the tip gap are properly captured. An O-grid that wraps the blade is added for allowing grid refinements closer to the blade surface. The O-grid has 35 grid points along the direction normal to blade surface. Grid refinements are also performed near the endwalls to resolve the turbulent boundary layer. The $y^{+}$at the walls is less than 2.

\section{Numerical Setup}

The steady, 3D compressible RANS equations are solved using a commercial code, ANSYS CFX 17.1. A high resolution upwind scheme (quasi second order) that blends the first and second order schemes is used for discretising the governing equations. This blended scheme addresses the shortcomings of the first and second order upwind schemes (ANSYS CFX 17.1 Documentation). For turbulence closure, a standard two equation $k-\epsilon$ model is chosen. The selection of the turbulence model is based on the outcome of the blind test study (Denton, 1997) where only the $k-\epsilon$ model managed to predict the correct hub pressure trend. Other researchers such as Hah and Loellbach (1999) and Shabbir et al. (1997) have successfully used the $k-\epsilon$ model for the Rotor 37 test case. A total pressure profile is prescribed at the inlet based on experimental data found in (Dunham, 1998). A simple radial equilibrium static 
pressure is imposed at the outlet. Stationary walls are set to counter-rotating to account for the relative motion since the domain is considered as a single domain in the rotating frame of reference. All walls are treated to be no slip, impermeable and adiabatic. Adiabatic walls are assumed due to unavailable experimental data although it has been shown by Bruna and Turner (2013) that the total temperature profile near the casing are better matched with isothermal conditions. The compressor map is constructed by gradually increasing the outlet static pressure from a value of $P_{2} / P_{a t m}=1.05$ (choking point). The stall point is determined iteratively such that any further increase in $\Delta P_{2}=5 \mathrm{~Pa}$ would no longer produce a solution that satisfies the following convergence criteria:

1) The calculations are run for a minimum of 2000 iterations.

2) Coefficient of variation (the ratio between the standard deviation to the mean) of the inlet mass flow rate value must not exceed 0.001 for the last 200 iterations.

3) Residuals for mass, momentum and energy for the last 1000 iterations behave normally. An algebraic multigrid (AMG) approach is implemented for accelerating the convergence rate of the solver (ANSYS CFX 17.1 Documentation). Figure 3 shows the example of the convergence history of a converged and non-converged simulation using the above criteria. For clarity, only the mass and CoV residuals are plotted.

Figure 3 a) Mass residuals and b) CoV history plot for a converged and non-converged case

\section{Validation}

For validation, the results obtained from the simulation are compared with the experimental data from Suder (1996). Figure 4 shows the performance map of the compressor compared against the experimental data. Total pressure ratio $(\pi)$ and adiabatic efficiency $(\eta)$ for operating point 4,6 and 8 fall in an error margin of $\pm 2 \%$ and $\pm 1 \%$, respectively. Operating point $4(\tilde{m}=0.983)$ and $8(\tilde{m}=0.927)$ are representative of the near design and near stall conditions as measured in the experiment, respectively. The mass flow rate, $m$, at operating point $1(\mathrm{~m}=20.97 \mathrm{~kg} / \mathrm{s})$ is the reference mass flow rate for obtaining the normalised mass flow rate, $\widetilde{m}$. The definitions for $\pi$ and $\eta$ are as obtained from Suder (1996). The simulation results agree well with the trend of the experimental result and especially in comparison to those reported in the blind test simulations described by Denton (1997). The numerical stall point is at a lower mass-flow than what was measured experimentally $(\tilde{m}=0.92)$ as shown in Figure 
3. The stall point is however subjective based on the backpressure increment step-size $\left(\Delta P_{2}\right)$ used to detect the last stable operating point.

Figure 4 Performance characteristics of Rotor 37 a) Total pressure ratio and b) Adiabatic efficiency

Figure 5 shows the pitchwise mass averaged total pressure radial distribution at the outlet for two representative operating points, near design, ND and near stall, NS. The simulation has managed to capture the trend of the pressure deficit at hub which was discussed by Denton (1997). The current authors believe that the total pressure deficit is likely due to a hub separation resulting from a potential hub leakage in the experiment as explained by Shabbir et al. (1997). The hub leakage is thought to have caused a reduced incidence angle locally that in the present simulation is mimicked by a rotating hub wall boundary condition upstream of the rotor. The pressure deficit near the hub was not present when the simulation was run with the rotor upstream hub held stationary.

Figure 5 Outlet radial distribution of $\pi$ for a) ND and b) NS

One important feature that needs to be validated for this study is the capability of the simulation to predict the shock location. This is important since a large error in the predicted location of the shock impacts on the blockage development that will be discussed later.

Figure 6 Pitchwise distribution of Relative Mach number at 95\% span and 20\% $c_{a x}$ a) ND and b) NS

Figure 6 shows the pitchwise Mach number profile for cases ND and NS at 95\% span inside the blade passage ( $20 \% c_{a x}$ ). The Mach number profile shows a good overall trend with the measured data despite under or over predicting the value of the 'trough'. This can be attributed to the weakness of the RANS simulation in predicting the shock-boundary layer interaction as explained by Denton (1997). Nevertheless, the simulation manages to capture the location of the 'trough' as what was measured in the experiment. The 'trough' which is located closer towards the blade pressure side at about 0.3 normalised pitch is due to deceleration of the flow behind the passage shock. The change in the depth of the 'trough' from 
ND to NS indicates a change in the passage shock structure and its interaction with the TLV. This can be seen in Figure 7 which presents the relative Mach number contour at 95\% span. The dashed line represents the location where the data was extracted to plot Figure 5.

Figure 7 Relative Mach number contour at 95\% span for a) ND and b) NS

The passage shock which extends throughout the span can be identified by the bunching of contour lines (near the blade LE) representing a sharp gradient in Mach number across the shock. From Figure 6, a change in the depth of the 'trough' occurs between the conditions ND and NS near 30\% blade pitch away from the pressure side. By examining Figure 7a) and Figure $7 b$ ), one can see that this is due to change in orientation, strength and location of the passage shock. Near stall, the Mach number downstream of the shock reduces drastically due to the blockage presented by the shock-TLV interaction. This is seen in Figure 7b) as a long dark region close to the blade pressure side downstream of the shock. At ND, the passage shock is attached to the blade and the shock angle almost aligned with the axial direction. At NS, the passage shock forms a bow shock where the shock no longer attaches to the blade and has moved upstream of the blade.

\section{Smooth Casing Results and Discussions}

\section{Shock-TLV Interaction}

The shock-TLV interaction is found to instigate TLV breakdown as the strength of the interaction increases (Yamada et al., 2007). The TLV breakdown creates a stagnation region inside the tip region which causes 'spillage'. 'Spillage' is the effect of blockage that diverts incoming flow to adjacent blade passages. Suder and Celestina (1996) found that at conditions near stall, the blockage region due to the shock-TLV interaction grows larger and moves upstream as the compressor is further throttled. In order to explain this matter, the normalised static pressure contour at $98 \%$ span is presented in Figure 8. The tip leakage vortex can be identified by the low pressure region extending from the tip LE. The tilting and upstream movement of the shock plane suggests that the point of interaction between the shock and TLV has also shifted. It can be noted from Figure $8 b$ ) that the shock interacts earlier and more strongly at conditions near stall as compared to conditions near design in Figure 8a). This further explains the upstream movement of the blockage region as noted by Suder and Celestina 
(1996). The increased blockage as a result of the stronger interaction near stall was described earlier using the Mach number contour plot in Figure 7b).

Figure 8 Static pressure contour at $98 \%$ span for a) ND and b) NS

\section{Radial Separation Vortex}

Figure 9 'Lambda-2' iso-surfaces near the casing

Another aerodynamic phenomenon that is responsible for blockage at the tip region is the radial separation vortex (RSV). The RSV has a tornado-like shape and its vortex line originates from the LE and ends at the casing. The theory behind the existence of this phenomenon is that the blade contains bound vortices that are responsible for lift. When the flow separates at the LE, the bound vortex is 'released or separated' from the blade to form a vortex with a predominantly radial component of vorticity. Using the 'lambda-2' criterion, coherent structures such as the tip leakage vortex and radial separation vortex in the tip region can be identified. The 'lambda-2' method works by locating a pressure minimum region by solving the eigenvalues of a Hessian matrix (Jeong and Hussain, 1995). Figure 9 shows the 'lambda-2' iso-surfaces at the casing region at NS. Two vortical structures; the TLV and the RSV are found to exist at the near casing region. Although Brandstetter and Schiffer (2018) identified it experimentally, to the best of the authors" knowledge, the RSV has never been proven to exist numerically in a transonic compressor.

The RSV is said to be responsible for a spike-type disturbance near the casing (Inoue et al., 1999; Pullan et al., 2015). It was shown by Inoue et al. (1999) that the RSV created an axial velocity deficit region near the casing downstream of the rotor blade. The rotation of the blade causes the RSV to stretch and break away from the blade and subsequently propagate into the adjacent blade passage. Within a low-speed compressor environment, Pullan et al. (2015) showed that the propagation of the RSV towards the adjacent blade generates a new separation and blockage region causing a sudden rise of static pressure upstream of the blade. This sudden rise in static pressure is found to be responsible the formation of a 'spike' type disturbance that will further develop into rotating stall. 


\section{Blockage}

The effect of blockage at the tip region ( $80 \%$ - 100\% span), can be visualised by plotting the normalised mass flux, $\tilde{\phi}$ contour at an axial plane located at $20 \% c_{a x, t}$ aft of the tip LE. The definition of $\tilde{\phi}$ is shown in Eq. 1 . Here, the quantity $\bar{\phi}$ is the mass-averaged value of mass flux, $\phi$, calculated across that plane.

$$
\widetilde{\phi}=\frac{\phi}{\bar{\phi}} \text { where } \phi=\rho V_{z}
$$

As shown in Figure 10a), the near casing flow is dominated by low $\tilde{\phi}$ which indicates blockage. In order to quantify the amount of blockage within the tip region, a blockage quantification method will be introduced. This method is an extension of the blockage identification method introduced in (Sakuma et al., 2013) to visualise blockage regions within the blade passage. The result of this blockage identification method calculated at an axial plane located at $20 \% c_{a x, t}$ aft of the tip LE is shown in Figure 10b) where only the 'blocked' cells are shown.

Figure 10 a) Normalised mass flux contour and b) 'Blocked' cells located at an axial plane of $20 \% c_{a x}$

The description of the blockage quantification method is as follows. At any given axial plane inside the domain, the mass flow rate crossing the plane must be equal to the inlet mass flow rate to satisfy continuity. The mass flow rate crossing that plane can be found by the summation of the mass flow rate across each cell contained in the plane. If a region with negative axial velocity exists, then the summation of all grid cells with positive mass flow must overshoot the inlet mass flow rate value before being balanced by the cells with negative axial velocity.

In order to define a blockage index $(\psi)$ the mass flow rate of cells in the plane are first sorted and summed in descending order. The summation will be stopped when the value of the sum equals the inlet mass flow rate. The remaining cells whose mass flow rate values are yet to be summed are considered as `blocked' cells.

'Blocked' cells will be assigned with value of, $\psi=1$ while un-'blocked' cells will be assigned a value of, $\psi=0$. By using this identification method, two blockage quantification parameters; non-dimensional blockage cell count $(\Psi)$ and non-dimensional blockage mass flow rate $\left(\Psi_{\mathrm{m}}\right)$ as in Eq. 2 and Eq. 3, respectively are introduced. 


$$
\begin{gathered}
\Psi=\frac{1}{\mathrm{~N}} \sum_{\mathrm{i}=1}^{\mathrm{N}} \psi(\mathrm{i}) \\
\Psi_{\mathrm{m}}=\frac{\sum_{\mathrm{i}=1}^{\mathrm{N}}|\psi(\mathrm{i}) \mathrm{m}(\mathrm{i})|}{\sum_{\mathrm{i}=1}^{\mathrm{N}} \mathrm{m}(\mathrm{i})}
\end{gathered}
$$

Here, $N$ is the total number of cells in the plane, $\psi(i)$ is the blockage index of the $i^{t h}$ cell and $m(i)$ is the mass flow associated with the $i^{\text {th }}$ cell. Note that in the evaluation presented here, each axial plane extends radially from 80 to $100 \%$ span.

Figure 11 Distribution of a) $\Psi$ and b) $\Psi_{m}$ across blade domain

Figure 11a) shows the axial distribution of non-dimensional blockage count $(\Psi)$ in the top $20 \%$ of span at the different operating points on the compressor map as in Figure 4a). The plot is obtained from 230 axial planes along the blade domain. As the compressor is 'throttled', the location of the peak blockage is seen to move up the span while also moving upstream. This is consistent with the upstream movement of the shock plane as the mass flow rate is lowered as described earlier. The non-dimensional blockage mass flow rate $\left(\Psi_{m}\right)$ as in Figure $11 \mathrm{~b}$ ) is found to peak at approximately $20 \% c_{a x, t}$ for the last converged point. The peak of the blockage has therefore moved forward by about $0.1 c_{a x, t}$ relative to ND.

\section{Optimisation Procedure}

As mentioned earlier, the aim of this study is to design a circumferential casing groove that improves the stability limit of the compressor. Previous SMI studies such as those by Shabbir and Adamczyk (2004) and Sakuma et al. (2013) have shown that circumferential casing grooves reduced the near casing blockage. This suggests that reducing the blockage near the casing could be the key to enhance the compressor stability limit. Accordingly, in this study the blockage distribution as shown in Figure 11 will form the basis of the first objective function $\left(f_{1}\right)$ that will be optimised. More accurately $f_{1}$ is the blockage count $(\Psi)$ located at the peak $\Psi_{m}$ at about $20 \% c_{a x, t}$. The second objective function $\left(f_{2}\right)$ used is the adiabatic efficiency $(\eta)$ of the tip region as evaluated over the $80-100 \%$ span region. A multi-objective optimisation 
routine is performed to find a groove design that reduces blockage by minimising $f_{1}$ while maximising $f_{2}$. The flow chart of the optimisation routine is shown in Figure 12.

Figure 12 Flow chart of optimisation procedure

Figure 13 a) Casing groove parameterisation and b) Example of LHS-generated grooves

Table 2 Casing groove design parameter limits

\section{Surrogate Model}

The role of the surrogate model is to reduce the computational cost by building an approximate model of high fidelity CFD simulations. The surrogate model used for this study is a supervised learning regression tree algorithm called Random Forest (RF) introduced by Breiman (2001). RF is an ensemble learning method where the output of the model is obtained from averaging the prediction of a set of regression trees. RF is an extended version of the 'bootstrapping aggregating (bagging)' method introduced also by Breiman (1996). 'Bagging' helps to overcome overfitting and hence improve the accuracy of the model by reducing the variance. 'Bootstrapping' is a sampling with replacement technique used to create multiple new samples of training data from the original training data set. This is to ensure that the trees generated are diverse and unique. For each new sample of training data, a regression tree is grown randomly using a recursive partitioning method (Cutler et al., 2012). A random predictor variable is chosen for each split to reduce the correlation between the trees. The split node is determined by finding the split that minimises the Residual Sum of Squares (RSS) as shown in Eq. 4. Here, $\mathrm{f}(\mathrm{x})_{i}$ and $\bar{f}(x)$ are the $i^{\text {th }}$ value of the training output and training output mean value, respectively. The output of the model is the average of the prediction value of all the trees hence the term 'aggregating'.

$$
\mathrm{RSS}=\sum_{\mathrm{i}=1}^{\mathrm{n}}\left(\mathrm{f}(\mathrm{x})_{\mathrm{i}}-\bar{f}(\mathrm{x})\right)^{2}
$$


The input training data is sampled using the Latin Hypercube Sampling (LHS) technique (Mckay et al., 2000). The LHS is a stratified sampling technique used to generate a quasi-random population of input variables across the design space. The LHS also ensures a good representation of all portions of the input variable design space. Table 2 shows the upper and lower bound of the casing groove design space. The input design parameters for the casing groove are shown in Figure 13a). The groove axial location $\left(z^{\prime}\right)$ and width $\left(w^{\prime}\right)$ are normalised by the tip axial chord $\left(c_{a x, t}\right)$. In this study, a total of 100 casing grooves are generated as shown in Figure 13b). The output training data are obtained by running the simulation of each of the 100 sampled grooved casing at the same conditions as operating point 8 in Figure 3 . The simulation is only performed at conditions same as operating point 8 as it is thought that the build-up of blockage at near stall conditions is responsible for the onset of stall. The output of the training data which are the objective functions, $f_{1}$ and $f_{2}$ are then evaluated for each of the simulation results for constructing the surrogate model.

\section{Performance of Random Forest}

One of the advantages of 'bagging' is that for each bootstrap sample data, there will be unused training data. The sampling with replacement procedure means that about a third of the training data will not appear in the bootstrap sample (Cutler et al., 2012). This unused training data set is referred as the 'out of bag' (OOB) data. The OOB data can be used for testing the predictive performance of the model since these data have not been used to train some of the trees in the model. For regression problems like the one in this study, the Mean Squared Error (MSE) as shown in Eq. 5 is used to estimate the generalisation error of the model. Here, $\mathrm{f}(\mathrm{x})_{i}$ is $i^{\text {th }}$ value of the training output and $\hat{f}(x)_{i}$ is the $i^{\text {th }}$ value of predicted value.

$$
\operatorname{MSE}=\frac{1}{n} \sum_{i=1}^{n}\left(f(x)_{i}-\hat{f}(x)_{i}\right)^{2}
$$

Figure $14 \mathrm{OOB}$ error of the surrogate model

Figure 14 shows the MSE of the model plotted against the number of regression trees generated. It can be seen that the MSE stabilises after the generation of a few hundred trees which suggests that a level of convergence has been achieved and that adding more trees will not affect the accuracy of the model. 


\section{Multi-Objective Genetic Algorithm}

The optimisation algorithm implemented in this study is a Multi-Objective Genetic Algorithm (MOGA). Genetic Algorithm (GA) is a random search method in optimisation that is inspired from the principle of natural selection (Goldberg, 1989). Calculus-based search methods such as direct methods operate by finding the steepest gradient to determine the optimum value. This requires the objective function to be smooth with a well-defined slope value. In reality, the objective functions are discrete due to errors and noise in the data. Random search methods are useful when dealing with non-differentiable objective functions since the search domain is randomly searched in the direction of the best possible solution. This is repeated until the optimum solution is found. This is one of the key advantages of GA when dealing with discontinuity in the data set.

GA performs three operations in its search method which are `selection', 'cross-over' and 'mutation'. 'Selection' is the process of finding the best possible parent (solution) using the principle of survival of the fittest. A 'tournament' is introduced between the randomly generated populations to search for the fittest individuals. The fitness of each individual is assessed by evaluating the objective function. The winners of the 'tournament' are then selected for 'cross-over'. 'Cross-over' mimics the reproduction process in creating a new set of generations. The genetic information of the parents are randomly selected and combined to create off-springs. A 'mutation' process is introduced to maintain the diversity of the population. 'Mutation' at a small rate protects the newly generated population from over-bias towards their ancestors. This is important in the search algorithm as it allows the exploration to continue without too much bias in any single direction. The GA algorithm routine will be stopped when the average relative change in the fitness of the generation reaches a certain tolerance.

Figure 15 Pareto front obtained from the optimisation routine

MOGA is introduced to solve multi-objective optimisation problems since GA was only meant for single objective problems. Fonseca and Fleming (1993) introduced a rank based method in assigning the fitness of the population. This rank method allows for MOGA to find a Pareto-optimal solution. A concept of dominance is introduced to rank the fitness of each individuals in a population. The rank of an individual is determined by the number of 
dominating individuals. A Pareto front is found when there exists a set of non-dominant individuals each with a rank equal to 1 .

\section{Result of Optimisation}

Figure 15 shows the Pareto-optimum solution obtained from the optimisation routine. A total of 18 optimum casing groove design inputs are obtained from the Pareto solution and the meridional cut of the geometries are shown in Figure 15.

Figure 16 Pareto solution of casing groove designs

All the groove designs are run through CFD to verify the optimisation results. The groove designs are run at the same backpressure as point 13(last stable operating point) in Figure 4. Only three converged cases are found as indicated by the filled circles in Figure 15. Among the converged cases, one casing groove design (highlighted in red in Figure 16) is selected for further verification by obtaining the performance curve and stall point. The design specifications of that optimum casing groove design is shown in Table 3.

$$
\begin{gathered}
\zeta=1-\frac{\pi_{\mathrm{ND}} \widetilde{\mathrm{m}}_{\mathrm{LAST}}}{\pi_{\mathrm{LAST}} \widetilde{\mathrm{m}}_{\mathrm{ND}}} \\
\Delta \zeta=\zeta_{\mathrm{GC}}-\zeta_{\mathrm{SC}}
\end{gathered}
$$

Table 3 Optimum casing groove parameters

Figure 17 Optimum groove performance map a) Total pressure ratio and b) Adiabatic efficiency

Figure 17 Optimum groove performance map a) Total pressure ratio and b) Adiabatic efficiency shows the performance curve for the selected optimum groove casing in comparison to the smooth casing. In order to compare the performance of the smooth and grooved casing, two parameters are considered, stall margin, $\zeta$ (Eq. 6) and stall margin improvement, $\Delta \zeta$ (Eq. 7). Here, $\Delta \zeta$ is the percentage change of the stall margin ( $\zeta)$ due to the casing groove. It is found that the groove increases the $\zeta$ by about $1 \%$. The magnitude of $\Delta \zeta$ gained here is not important as the aim of this study is to show that an optimised groove that improves the $\Delta \zeta$ can be obtained by optimising for blockage. In addition, $\Delta \zeta$ is relative to the numerical scheme used and also the method of finding the last stable operating point. A fair comparison with respect to other casing treatment studies using Rotor 37 as a test case can only be made if the 
same numerical scheme and convergence criteria is used. Furthermore, as shown in Figure 16b), the overlapping between the $\eta$ curves show that the $\eta$ penalty due to the groove is within $\pm 0.1 \%$ in comparison to the smooth casing.

Currently, known methods to find the best performing groove such as those by Houghton and Day (2010) and Sakuma et al. (2013) use a trial and error approach whereas in this study a physics based approach is attempted. The result of this optimisation method shows the reliability of using a blockage parameter definition as the objective function when searching for a casing groove that improves the stability of a compressor.

\section{Effect of groove on blockage}

The effect of the groove on the blockage is described by comparing the $\Psi_{m}$ distribution, for the top $20 \%$ of span, for smooth and grooved casing as shown in Figure 18. The shaded region represents the influence of the groove on the $\Psi_{m}$ distribution. Far upstream and downstream of the blade, the $\Psi_{m}$ distribution for both cases are nearly identical. Although the groove is only $5 \% c_{a x, t}$ in width and is located at $20 \% c_{a x, t}$ downstream of the LE the reduction in blockage due to it starts at $10 \% c_{a x, t}$ downstream of the LE and up to the middle of the groove. The maximum blockage reduction is seen to coincide with the LE location of the groove. The blockage is then found to increase and peak at the TE of the groove. The reduction of blockage due to the groove was clearly explained by Sakuma et al. (2013) since the groove allowed for radial transport of fluid. Figure 19 shows the Mach number contour inside the tip gap region for both smooth and groove casing. Blockage can be identified as a dark region with Mach number less than 0.2. The shape of the blockage distribution has changed for the grooved casing as compared to the smooth casing. The blockage region for the grooved casing is split with the bulk of the blockage now located further downstream of the groove. The groove is therefore able to re-distribute the blockage from front to aft and also cause an overall reduction in blockage in the tip gap region both of which are thought to be responsible for the stall margin improvement. This blockage based analysis shows that the stalling mass flow is sensitive not only to the amount of blockage in the tip region but also its chord-wise location as the compressor is seen to be able to withstand higher blockages down the chord.

Figure 18 Comparison of $\Psi_{m}$ at point 8(NS) for smooth and grooved casing

Figure 19 Mach number contour for a) smooth and b) groove casing inside the tip gap 


\section{Effect at Part-speed}

At part-speed condition (60\% of design speed), the flow is reported to be free of passage shock (Suder and Celestina, 1996). This allows for the evaluation of the performance of the groove when the near casing blockage is no longer affected by the shock-TLV interaction. The calculation however do not account for the changes in blade twist and tip gap height due to the reduction in centrifugal force at part-speed (Suder and Celestina, 1996). Thus, the result presented herein is solely intended for evaluating the groove performance at part-speed and is not comparable to the actual experimental data. Figure 20 shows the performance map at partspeed for smooth and grooved casing. It can be seen that the neither the efficiency $(\eta)$ or the stall margin $(\zeta)$ is detrimentally affected by the groove. At part speed, the groove gives an SMI improvement $(\Delta \zeta)$ of about $1 \%$ using the same definition as in Eq. 7.

Figure 20 Performance characteristics at part-speed a) Total pressure ratio and b) Adiabatic efficiency

The reason for the SMI improvement can be understood by plotting the $\Psi_{m}$ distribution, for the top $20 \%$ of span inside the blade passage for a few operating points as shown in Figure 21. The peaks of the blockage at different operating points show the development of the blockage as the compressor is throttled. Without the influence of shock, it can be seen that the forward movement of the peak blockage location (when throttled) is not as pronounced as in the high speed case. The blockage at peak is also seen to be more distributed in the chord-wise direction under part-speed conditions as a shock mechanism no longer exists. The peak of the blockage is located closer to the LE and increases in height at conditions closer to stall. It can be seen that the blockage for every operating point shown is affected by the groove except for point 3 where the blockage is relatively small and does not extend to the chord-wise location of the groove. The effect of the groove is to push the blockage downstream, i.e. closer to it, and thus allowing a little more room to reduce mass flow further before stalling. It seems to suggest that, for each speed condition, there exists a limiting forward location for the blockage peak to exist, beyond which the compressor would undergo stall. On comparing Figure 11 and Figure 21 one can see that, near stall, the compressor at $60 \%$ speed is able to withstand more than double the peak blockage as endured when at $100 \%$ speed. The location of the peak blockage can also be seen to be located further upstream at part speed $\left(10 \% c_{a x, t}\right)$ than for the $100 \%$ speed $\left(21 \% c_{a x, t}\right)$ case showing that the compressor is more sensitive to the blockage at higher operating speeds. 
Figure 21 Comparison of $\Psi_{m}$ distribution for smooth and grooved casing

\section{Conclusions}

A steady RANS simulation was performed and validated to understand the near casing flow phenomena that are responsible for aerodynamic blockage in a transonic axial compressor. The performance characteristics of the numerical simulation agrees well with the experimental data where the error margin for $\pi$ and $\eta$ were within $\pm 2 \%$ and $\pm 1 \%$, respectively. The simulation also managed to capture important flow features such as the passage shock location and outlet hub pressure deficit that were reported in the experiments. The conclusions of the present study are summarised as the following:

1. The shock-TLV interaction and RSV were identified as the source of blockage near the casing. At conditions close to the stall limit, the shock detaches from the blade and moves upstream. The movement and detachment of the shock from the blade causes the shock to interact more strongly with the TLV. The upstream movement of the shock results in the early interaction between the shock and TLV which subsequently causes the blockage to move upstream.

2. The RSV was observed to exist in a transonic rotor environment by using a vortex identification method, 'lambda-2'. To the authors' best knowledge, RSV has never been reported to exist in Rotor 37 so far. The existence of the RSV suggests that it is partly responsible for generating blockage near the casing.

3. The near casing blockage can be visualised by plotting the non-dimensional mass flux, $\tilde{\phi}$, contour. Low $\tilde{\phi}$ regions are found to dominate the near casing region as a result of the shock-TLV interaction and RSV. A blockage index $(\psi)$ was introduced to identify 'blocked' cells and this method has been extended to quantify blockage across the blade domain. The axial distribution of the non-dimensional blockage count $(\Psi)$ shows that at conditions close to stall, the peak of the distribution moves upstream. The nondimensional blockage mass flow $\left(\Psi_{m}\right)$ shows that the blocked mass flow rate for the last converged point peaks at about $20 \%$ of the $c_{a x, t}$ aft of the tip LE. The blockage analysis has helped to highlight the sensitivity of the compressor to the blockage amount and location along the chord and how this sensitivity changes when operated at part speed.

4. A surrogate-based optimisation procedure was employed to find an optimum casing groove design for SMI. A MOGA technique was used to search for the optimum casing 
design using the blockage analysis obtained from the smooth casing results. The optimiser searches for the casing groove that minimises the blockage at about $20 \%$ of the $c_{a x, t}$ aft of the tip LE with the least efficiency penalty. Using this method, an optimum groove design was obtained and it is found that the groove improves the stall margin by about $1 \%$. At part-speed conditions the groove is found to have no detrimental effects to the stall margin and efficiency. This shows that instead of a trial and error approach, the axial location and sizing of the groove can be obtained, numerically, through the optimisation of an appropriately defined blockage parameter.

5. The computational cost of the optimisation has been reduced in two different ways. First, the use of the surrogate model reduces the cost of the optimiser algorithm for evaluating the objective function. The surrogate model replaces the need for using highfidelity CFD simulations when finding the optimum groove design. Secondly, the use of a blockage parameter as the objective function instead of SMI has reduced the number of operating points that are needed to be numerically simulated for each candidate groove geometry. The optimisation is performed at only one operating point (near stall condition) since it is the build-up of blockage near stall that has shown to be responsible for stall.

\section{Nomenclature}

Symbols

c chord

f Objective function

$\mathrm{H}$ Groove Height

m Mass flow rate

$\mathrm{N}$ Total number of cells in a plane

P Pressure

s span

V Velocity

$\mathrm{w}^{\prime} \quad$ Normalise groove width

$\mathrm{z} \quad$ Axial direction

$\mathrm{z}^{\prime} \quad$ Normalise axial location of groove

$\Delta \zeta \quad$ Stall Margin Improvement (SMI)

$\zeta \quad$ Stall margin 


$\begin{array}{ll}\eta & \text { Adiabatic efficiency } \\ \pi & \text { Total pressure ratio } \\ \Omega & \text { Blade speed } \\ \phi & \text { Mass flux } \\ \rho & \text { Density } \\ \psi & \text { Blockage index } \\ \Psi & \text { Blockage parameter }\end{array}$

Subscripts

$\begin{array}{ll}\text { atm } & \text { Atmospheric } \\ 0 & \text { Total } \\ 1 & \text { Inlet } \\ 2 & \text { Outlet } \\ \text { ax } & \text { axial } \\ \text { LAST } & \text { Last operating point } \\ \text { GC } & \text { Groove casing } \\ \text { SC } & \text { Smooth casing } \\ \text { t } & \text { tip } \\ \text { z } & \text { Axial direction } \\ \text { Operators } & \\ \sim & \text { Normalised } \\ - & \text { Averaged } \\ \wedge & \text { Predicted }\end{array}$

\section{References}

ANSYS CFX 17.1 Documentation

Brandstetter, C. and Schiffer, H.-P. (2018), "PIV measurements of the transient flow structure in the tip region of a transonic compressor near stability limit", Journal of the Global Power and Propulsion Society, Vol. 2, pp. 303-316.

Breiman, L. (1996), “Bagging predictors”, Machine Learning, Vol. 24 No. 2, pp. 123-140.

Breiman, L. (2001), “Random Forests”, Machine Learning, Vol. 45 No. 1, pp. 5-32. 
Bruna, D., and Turner, M. G. (2013). "Isothermal Boundary Condition at Casing Applied to the Rotor 37 Transonic Axial Flow Compressor”, Journal of Turbomachinery, Vol. 135 No.3, 034501.

Cutler, A., Cutler, D.R. and Stevens, J.R. (2012), "Random Forests”, in Zhang, C. and Ma, Y. (Eds.), Ensemble Machine Learning, Springer US, Boston, MA, pp. 157-175.

Denton, J.D. (1997), “Lessons from rotor 37”, Journal of Thermal Science, Vol. 6 No. 1, pp. $1-13$.

Dunham, J. (1998), “CFD Validation for Propulsion System Components (la Validation CFD des organes des propulseurs)", p. 95.

Fonseca, C.M. and Fleming, P.J. (1993), “Genetic Algorithms for Multiobjective Optimization: FormulationDiscussion and Generalization", Proceedings of the 5th International Conference on Genetic Algorithms, Morgan Kaufmann Publishers Inc., San Francisco, CA, USA, pp. 416-423.

Furukawa, M., Inoue, M., Saiki, K. and Yamada, K. (1999), “The Role of Tip Leakage Vortex Breakdown in Compressor Rotor Aerodynamics”, Journal of Turbomachinery, Vol. 121 No. 3, pp. 469-480.

Goldberg, D.E. (1989), Genetic Algorithms in Search, Optimization and Machine Learning, 1st ed., Addison-Wesley Longman Publishing Co., Inc., Boston, MA, USA.

Hah, C., and Loellbach, J. (1999), "Development of Hub Corner Stall and Its Influence on the Performance of Axial Compressor Blade Rows." Journal of Turbomachinery, Vol. 121 No. 1, pp. 67-77.

Hathaway, M.D. (2007), Passive Endwall Treatments for Enhancing Stability, NASA Report No. TM-2007-214409, NASA. 
Houghton, T. and Day, I. (2010), "Enhancing the Stability of Subsonic Compressors Using Casing Grooves”, Journal of Turbomachinery, Vol. 133 No. 2, pp. 021007-02100711.

Inoue, M., Kuroumaru, M., Tanino, T. and Furukawa, M. (1999), "Propagation of Multiple Short-Length-Scale Stall Cells in an Axial Compressor Rotor”, Journal of Turbomachinery, Vol. 122 No. 1, pp. 45-54.

Jeong, J. and Hussain, F. (1995), “On the identification of a vortex”, Journal of Fluid Mechanics, Vol. 285 No. 1, p. 69.

Mckay, M.D., Beckman, R.J. and Conover, W.J. (2000), “A Comparison of Three Methods for Selecting Values of Input Variables in the Analysis of Output from a Computer Code", Technometrics, Vol. 42 No. 1, p. 55.

Pakatchian, M.R., Saeidi, H. and Ziamolki, A. (2019), "CFD-based Blade Shape Optimization of MGT-70(3) Axial Flow Compressor", International Journal of Numerical Methods for Heat \& Fluid Flow, available at:https://doi.org/10.1108/HFF10-2018-0603.

Pullan, G., Young, A.M., Day, I.J., Greitzer, E.M. and Spakovszky, Z.S. (2015), “Origins and Structure of Spike-Type Rotating Stall”, Journal of Turbomachinery, Vol. 137 No. 5, pp. 051007-051007-11.

Sakuma, Y., Watanabe, T., Himeno, T., Kato, D., Murooka, T. and Shuto, Y. (2013), "Numerical Analysis of Flow in a Transonic Compressor With a Single Circumferential Casing Groove: Influence of Groove Location and Depth on Flow Instability”, Journal of Turbomachinery, Vol. 136 No. 3, p. 031017.

Shabbir, A., Celestina, M. L., Adamczyk, J. J., and Strazisar, A. J. "The Effect of Hub Leakage Flow on Two High Speed Axial Flow Compressor Rotors." Proceedings of the ASME 1997 International Gas Turbine and Aeroengine Congress and 
Exhibition. Volume 1: Aircraft Engine; Marine; Turbomachinery; Microturbines and Small Turbomachinery. Orlando, Florida, USA. June 2-5, 1997.

Shabbir, A. and Adamczyk, J.J. (2004), "Flow Mechanism for Stall Margin Improvement due to Circumferential Casing Grooves on Axial Compressors", Journal of Turbomachinery, Vol. 127 No. 4, pp. 708-717.

Simpson, T.W., Mauery, T.M., Korte, J. and Mistree, F. (2001), "Kriging models for global approximation in simulation-based multidisciplinary design optimization", AIAA Journal, Vol. 39, pp. 2233-2241.

Suder, K.L. (1996), Experimental Investigation of the Flow Field in a Transonic, Axial Flow Compressor with Respect to the Development of Blockage and Loss, PHD Thesis, Case Western Reserve University, Cleveland, Ohio, USA, October.

Suder, K.L. and Celestina, M.L. (1996), "Experimental and Computational Investigation of the Tip Clearance Flow in a Transonic Axial Compressor Rotor", Journal of Turbomachinery, Vol. 118 No. 2, pp. 218-229.

Yamada, K., Funazaki, K. and Furukawa, M. (2007), "The Behavior of Tip Clearance Flow at Near-Stall Condition in a Transonic Axial Compressor Rotor", Volume 6: Turbo Expo 2007, Parts A and B, presented at the ASME Turbo Expo 2007: Power for Land, Sea, and Air, ASME, Montreal, Canada, pp. 295-306. 




Figure 1 Meridional cut-view of NASA Rotor 37

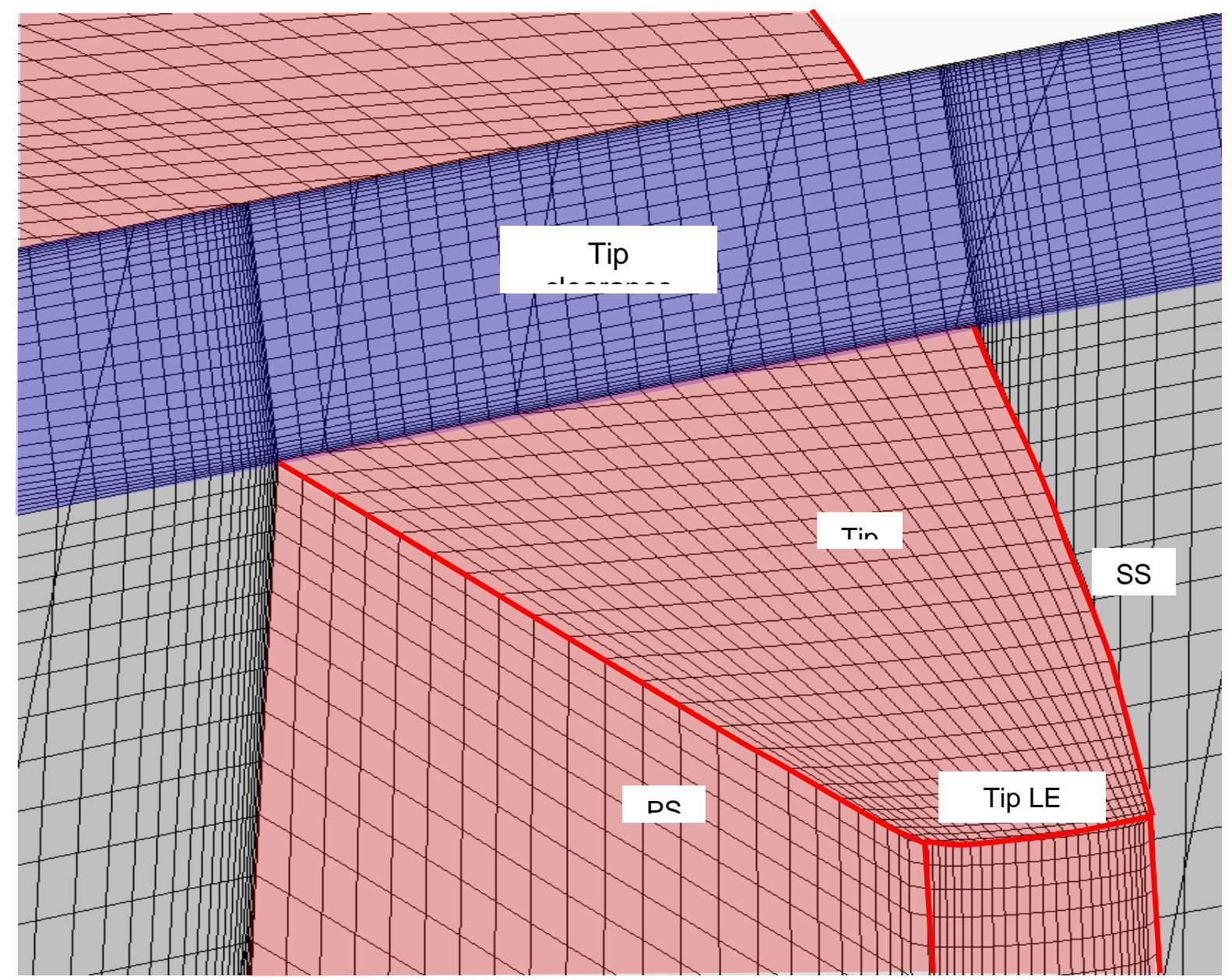

Figure 2 Grid points generated in the tip region 

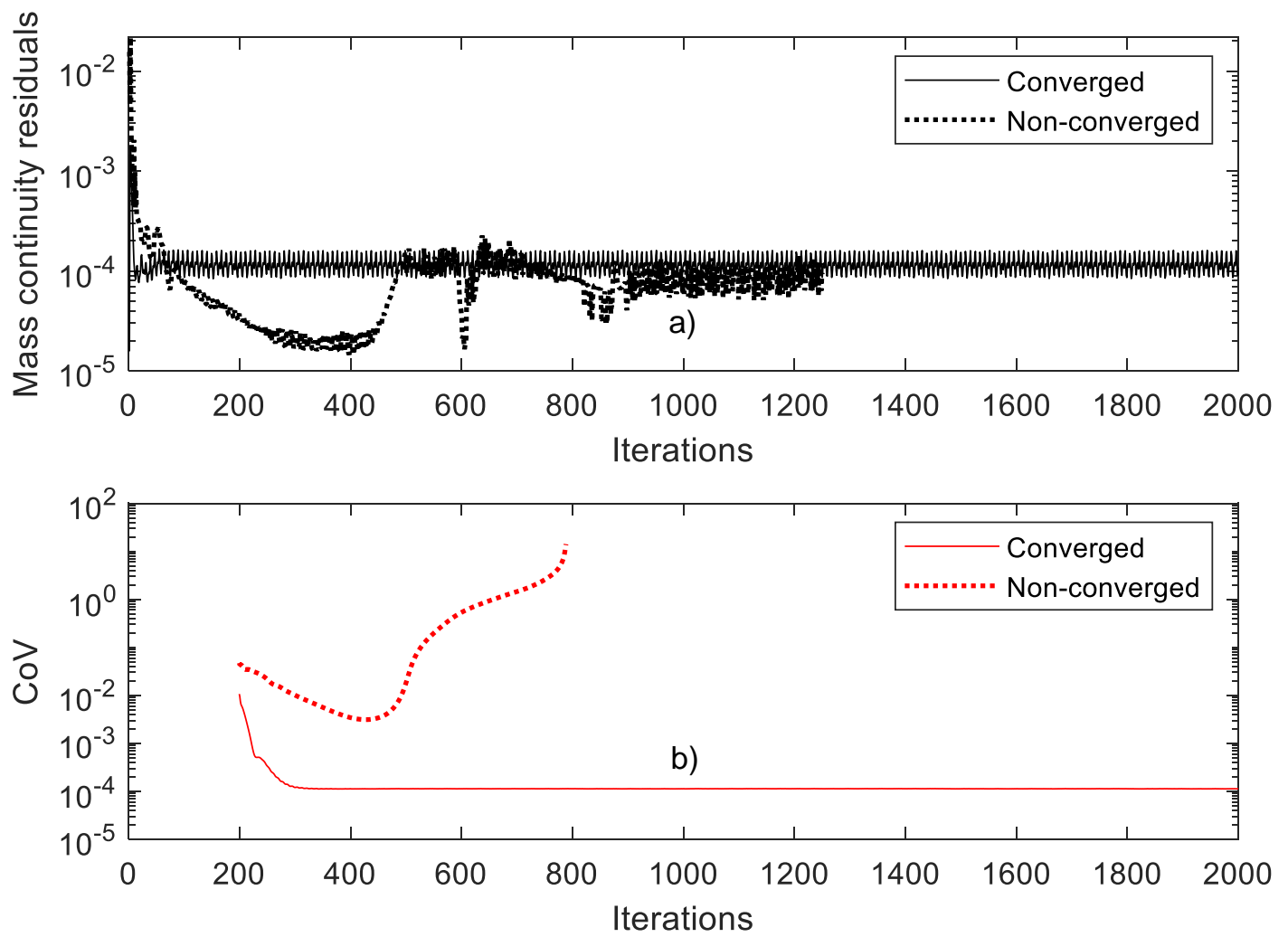

Figure 3 a) Mass residuals and b) CoV history plot for a converged and non-converged case
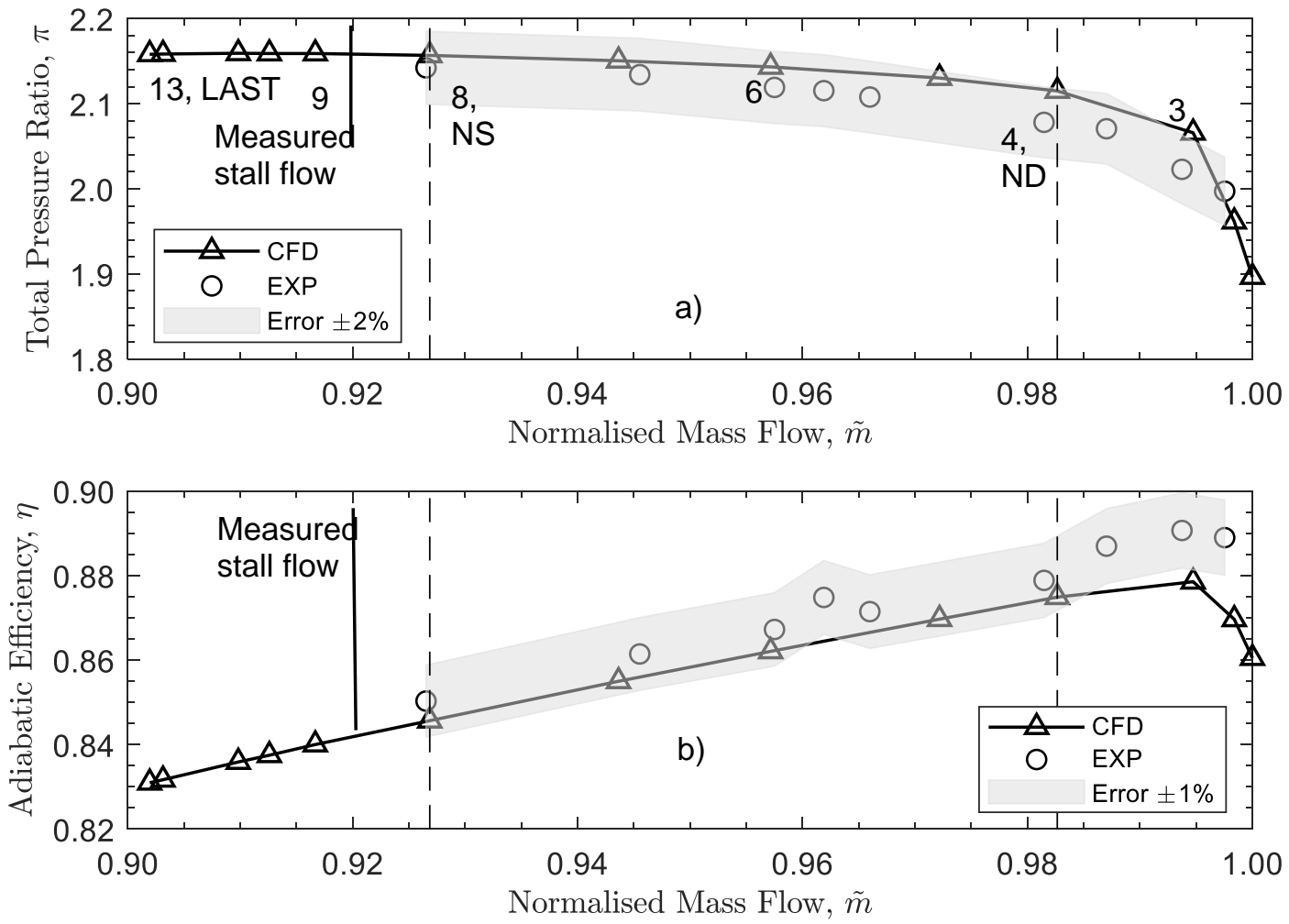

Figure 4 Performance characteristics of Rotor 37 a) Total pressure ratio and b) Adiabatic efficiency 

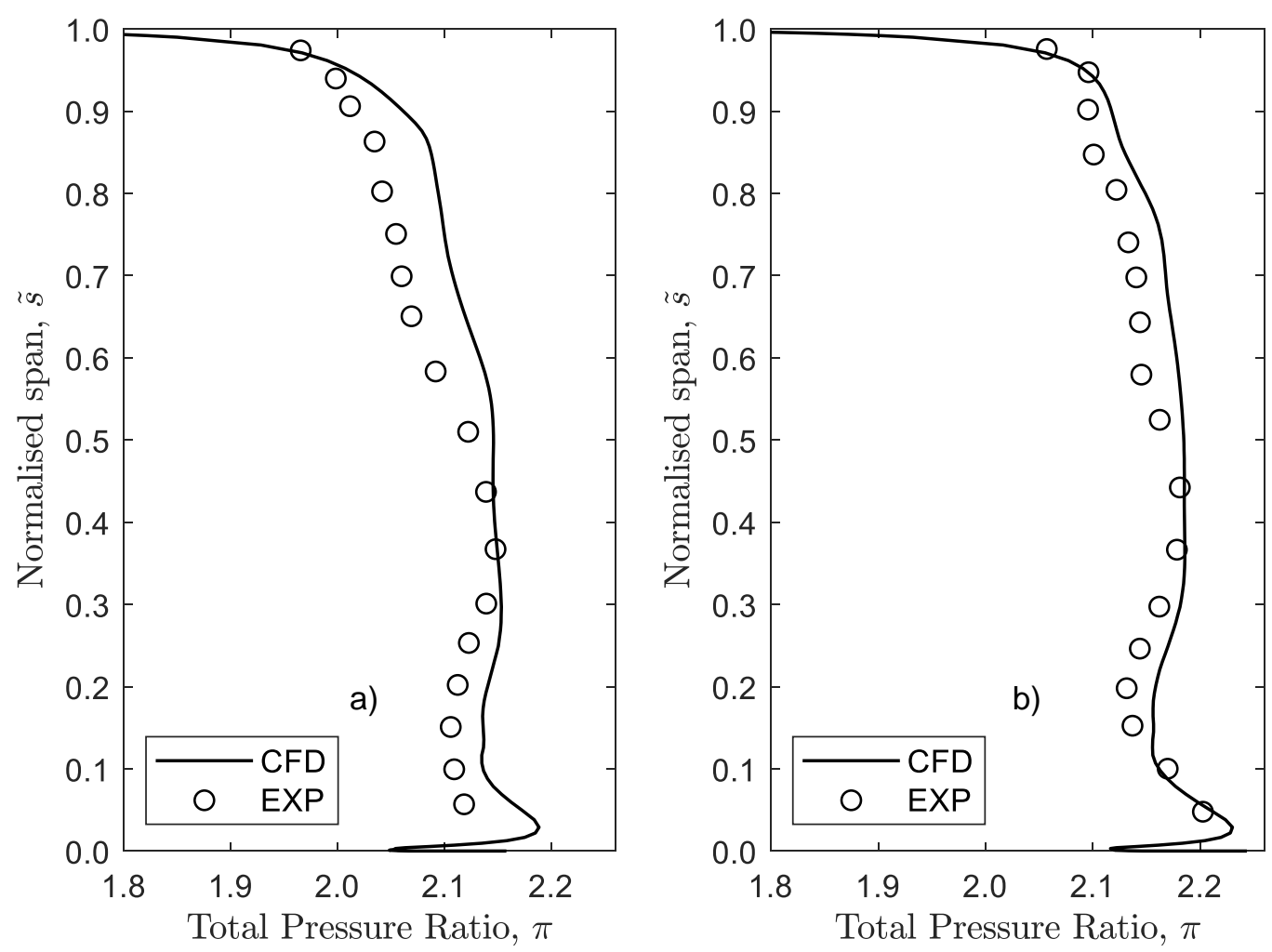

Figure 5 Outlet radial distribution of $\pi$ for a) ND and b) NS
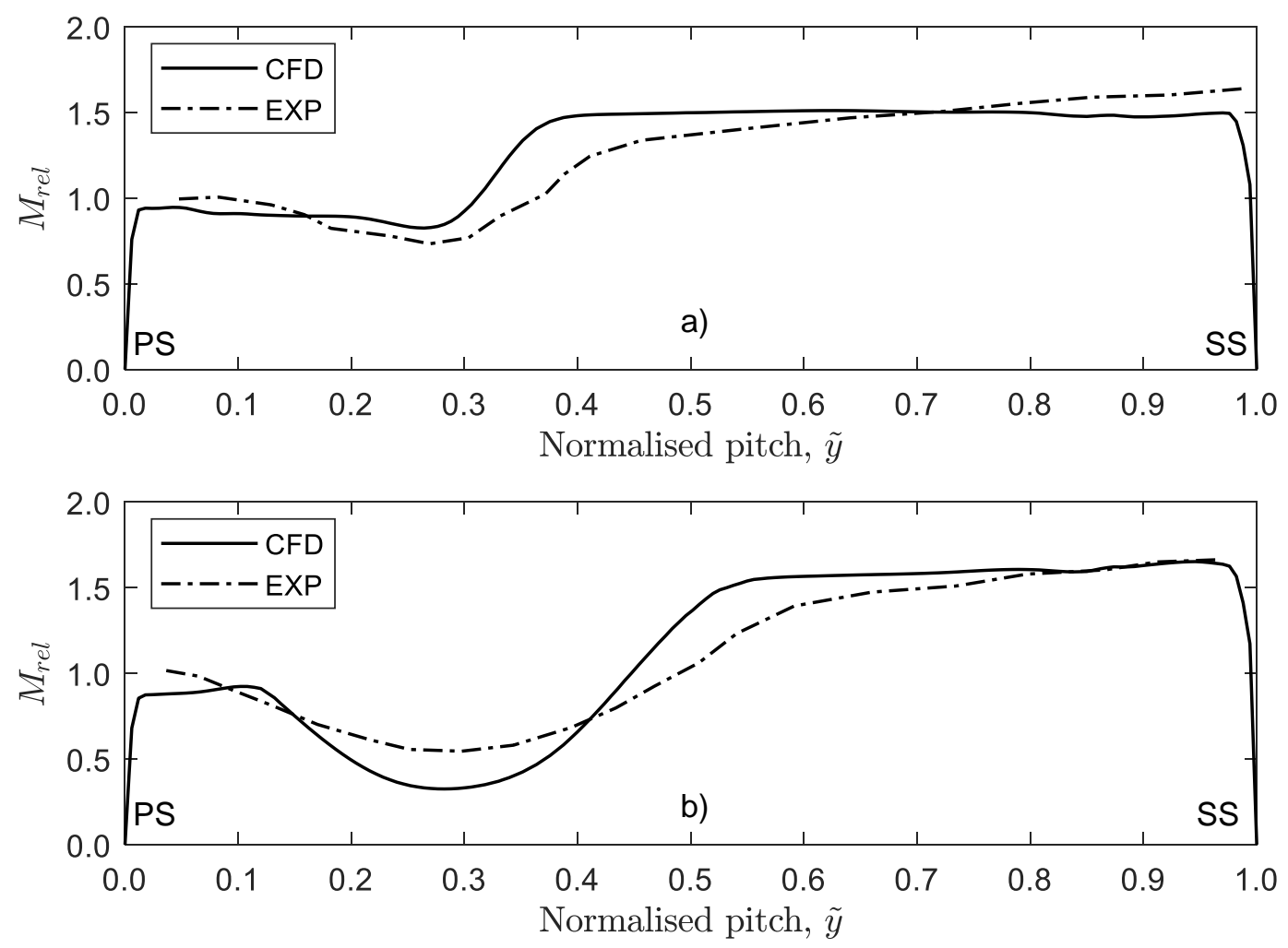

Figure 6 Pitchwise distribution of Relative Mach number at 95\% span and 20\% $c_{a x}$ a) ND and b) NS 

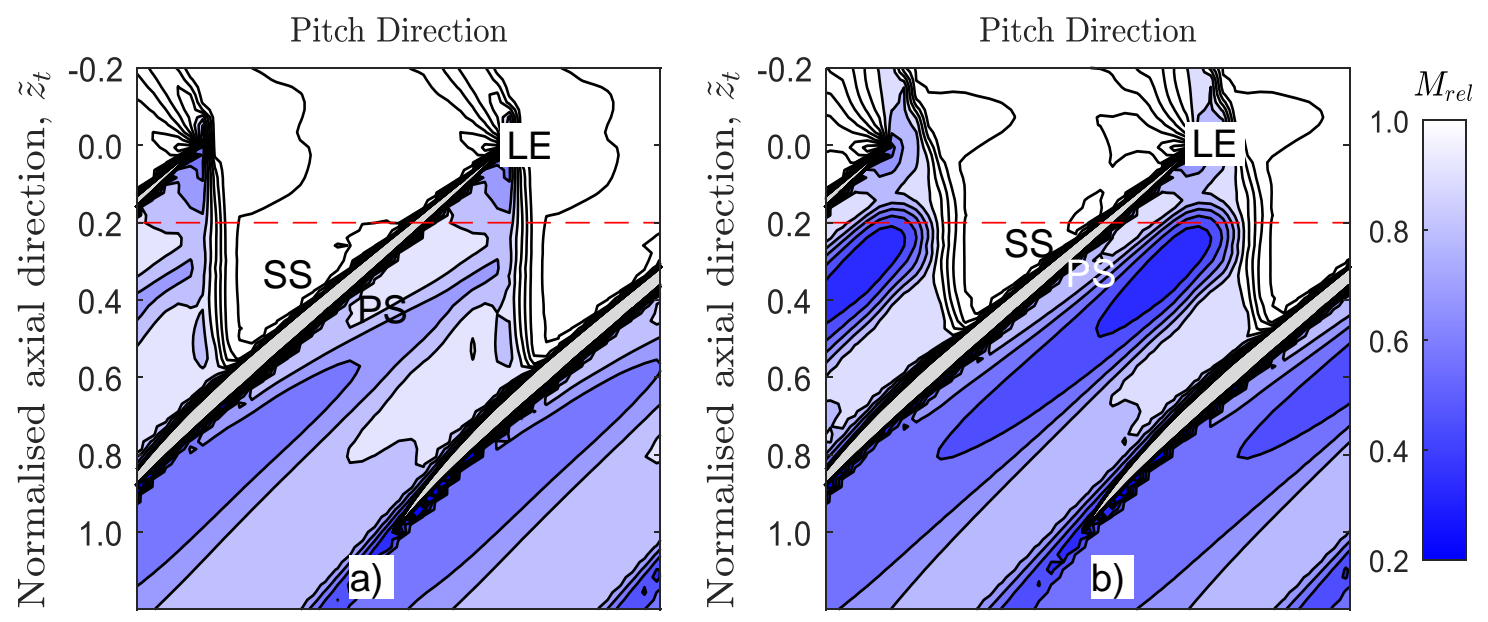

Figure 7 Relative Mach number contour at 95\% span for a) ND and b) NS
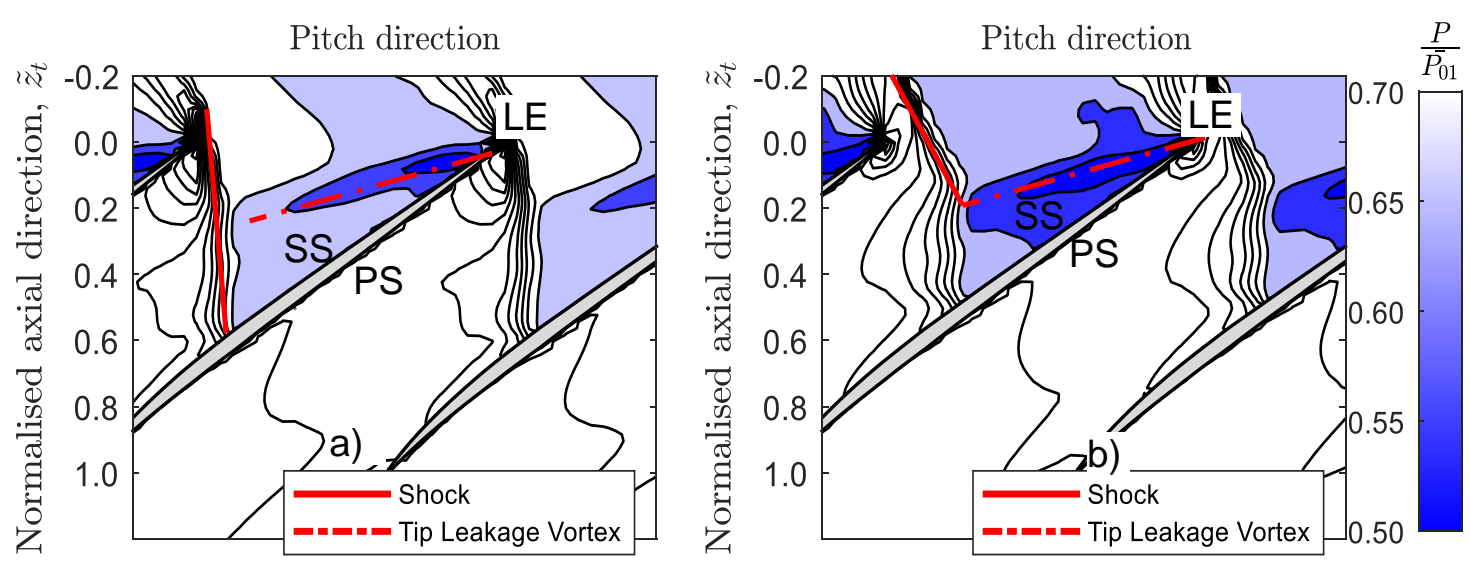

Figure 8 Static pressure contour at $98 \%$ span for a) ND and b) NS

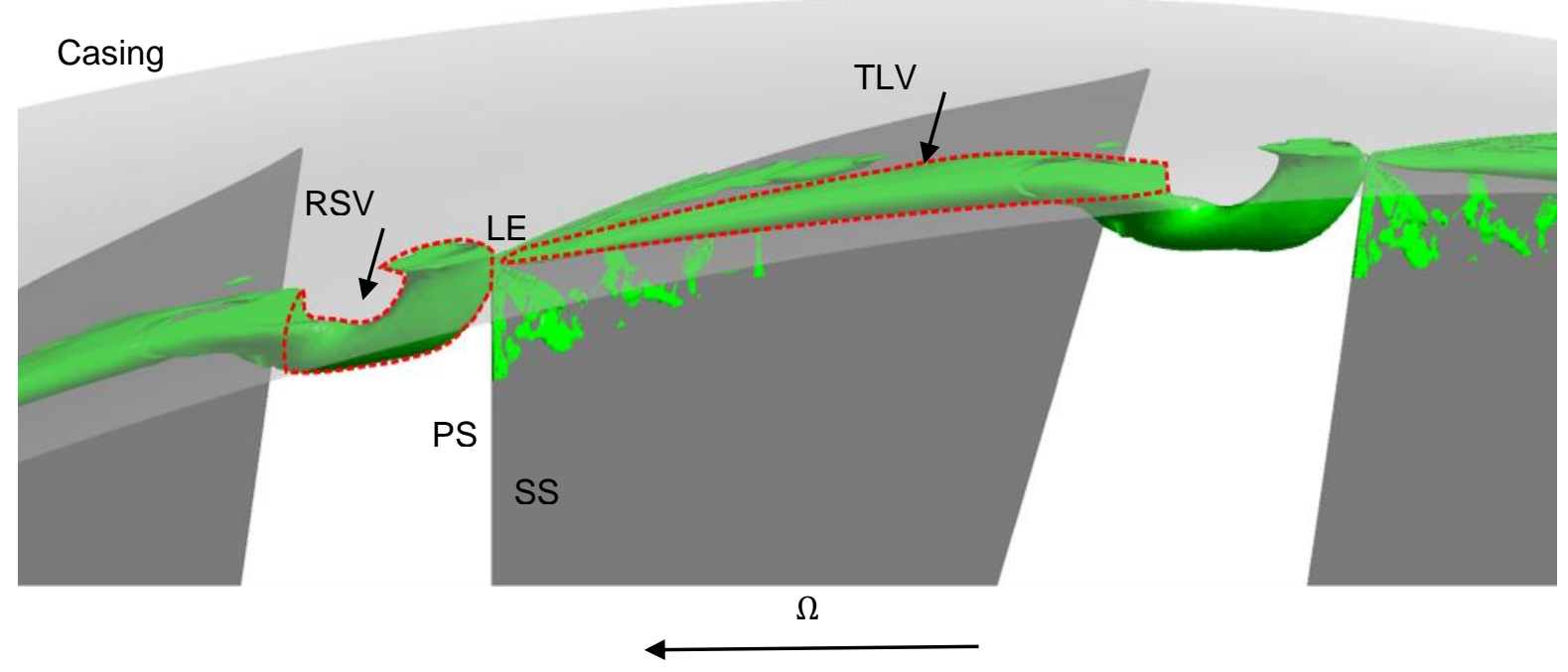

Figure 9 'Lambda-2' iso-surfaces near the casing 


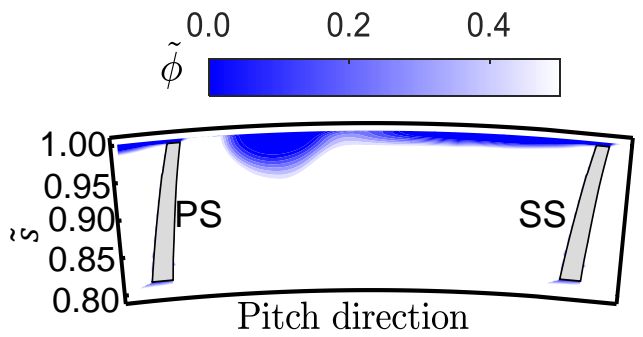

a)

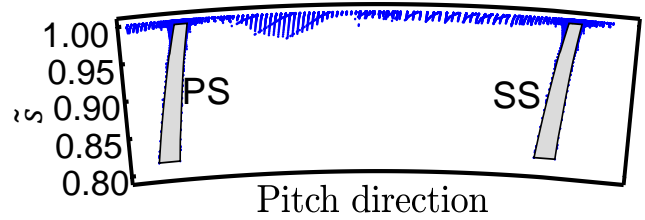

b)

Figure 10 a) Normalised mass flux contour and b) 'Blocked' cells located at an axial plane of $20 \% c_{a x}$
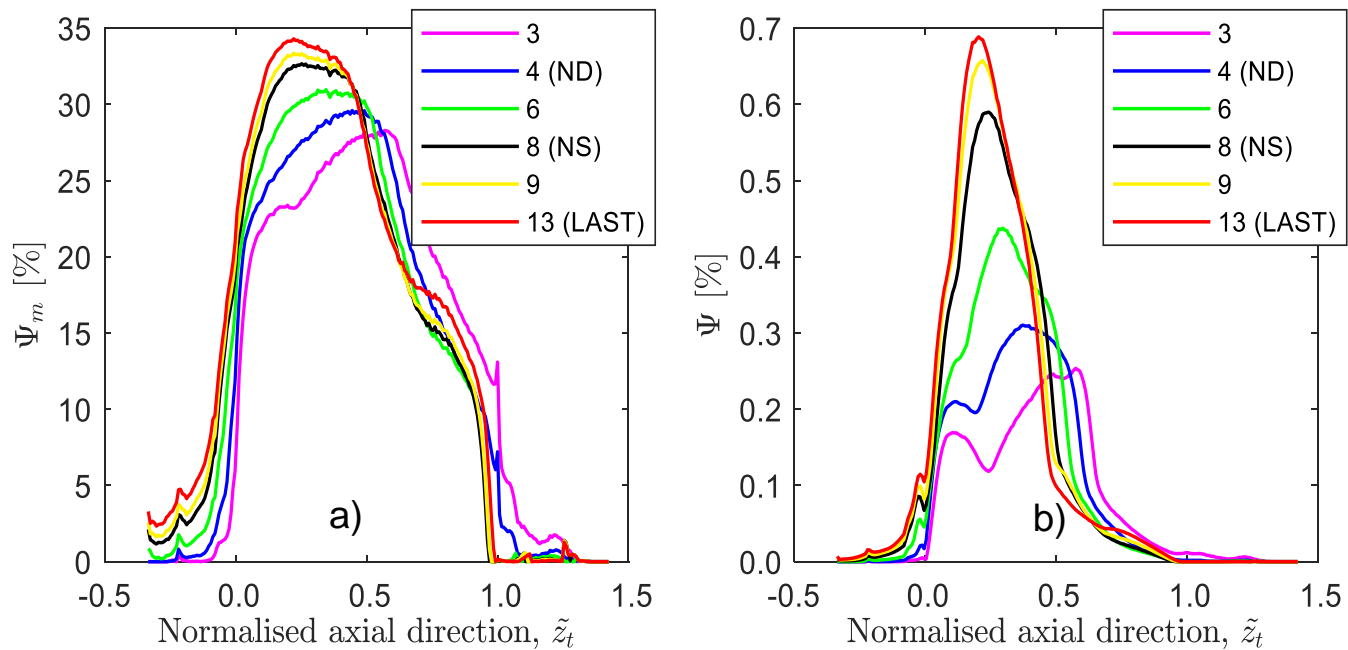

Figure 11 Distribution of a) $\Psi$ and b) $\Psi_{m}$ across blade domain 


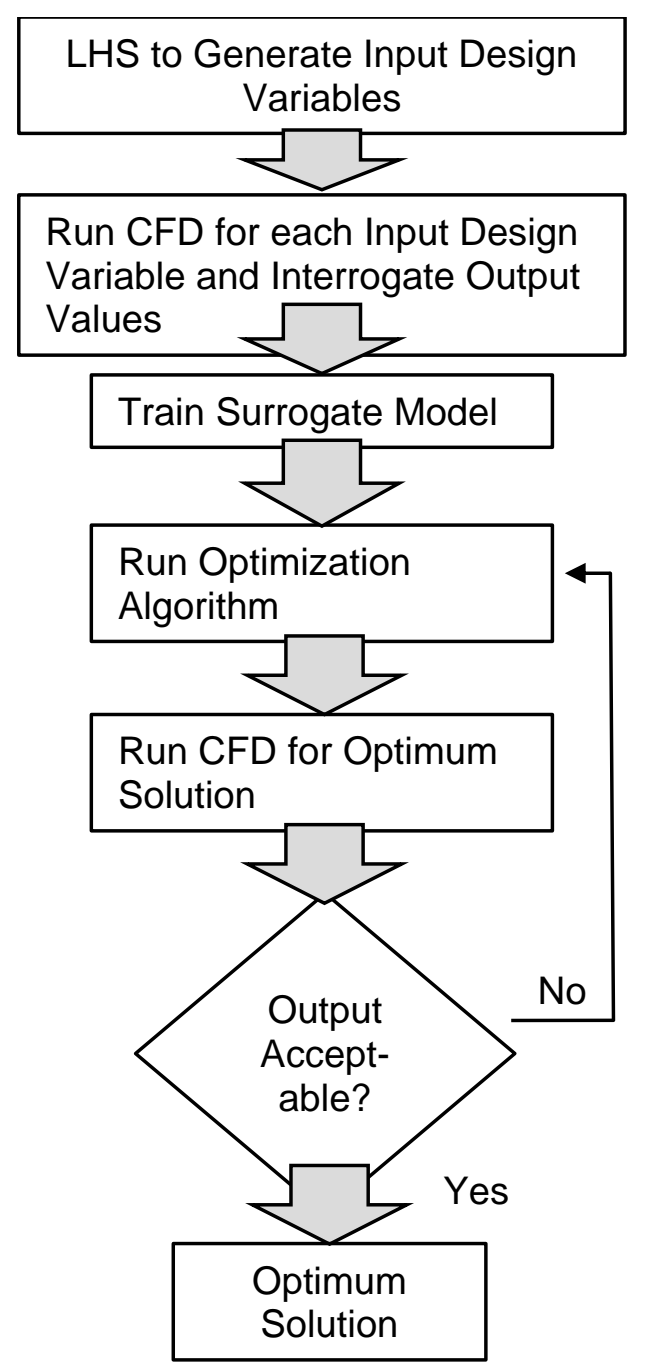

Figure 12 Flow chart of optimisation procedure

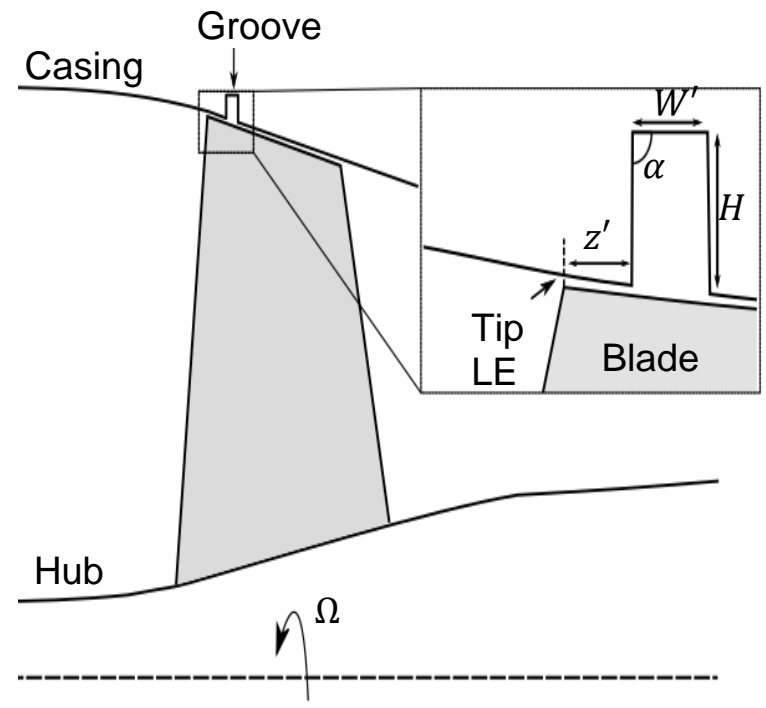

a)

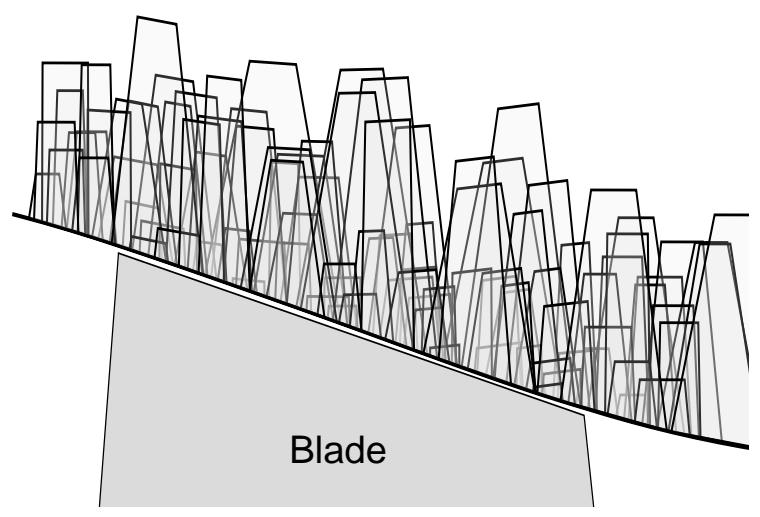

b)

Figure 13 a) Casing groove parameterisation and b) Example of LHS-generated grooves 


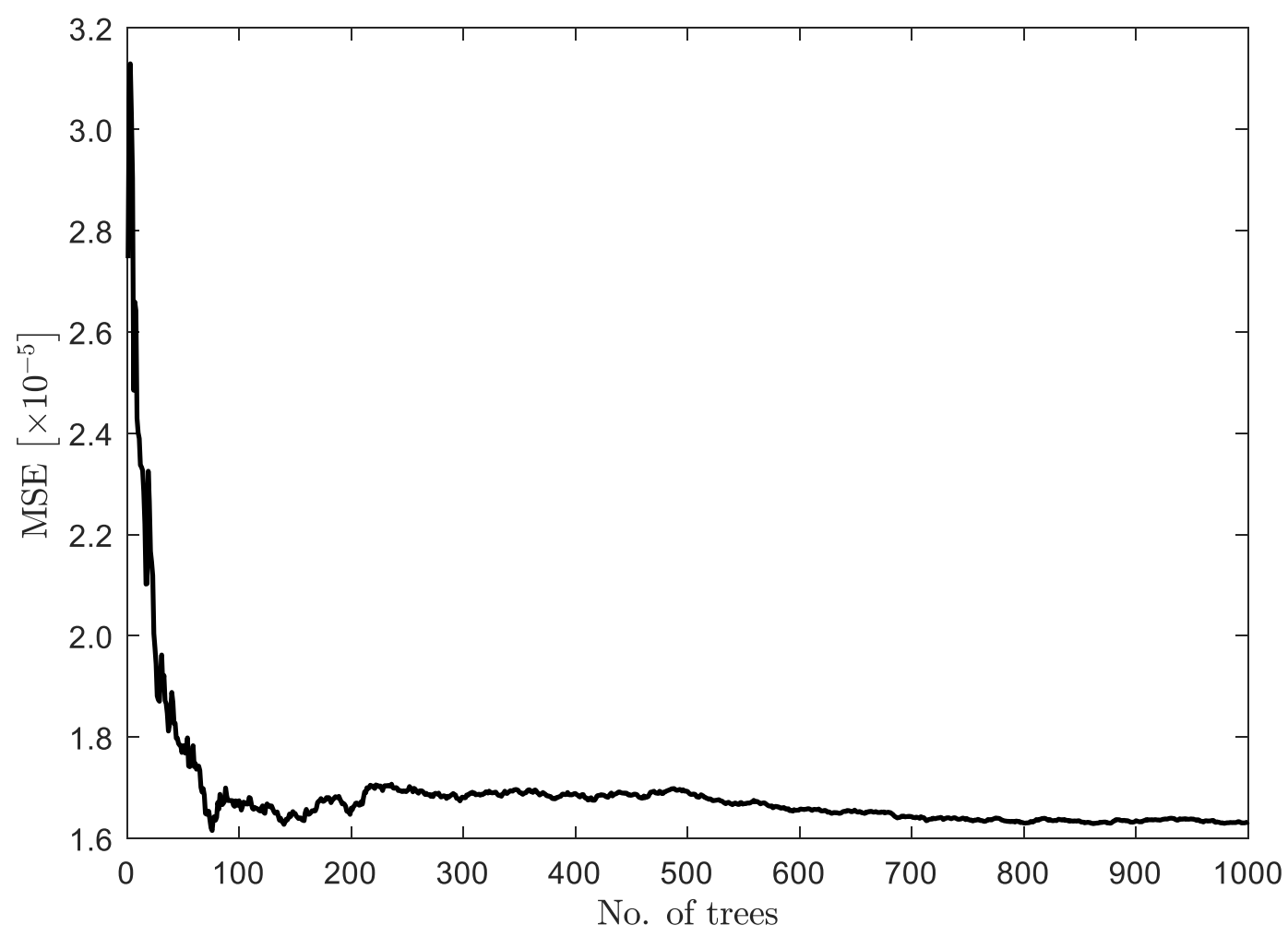

Figure 14 OOB error of the surrogate model

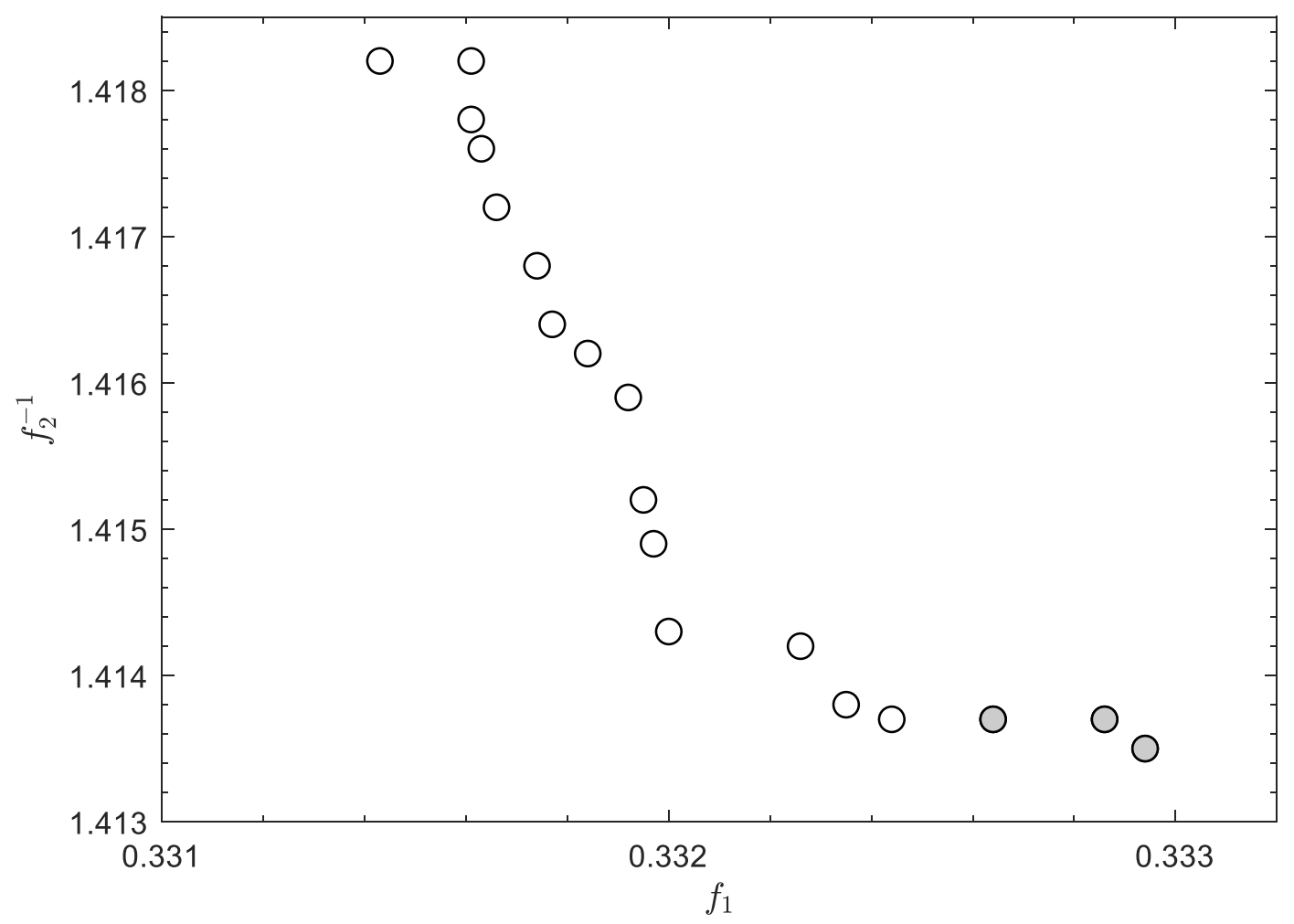

Figure 15 Pareto front obtained from the optimisation routine 


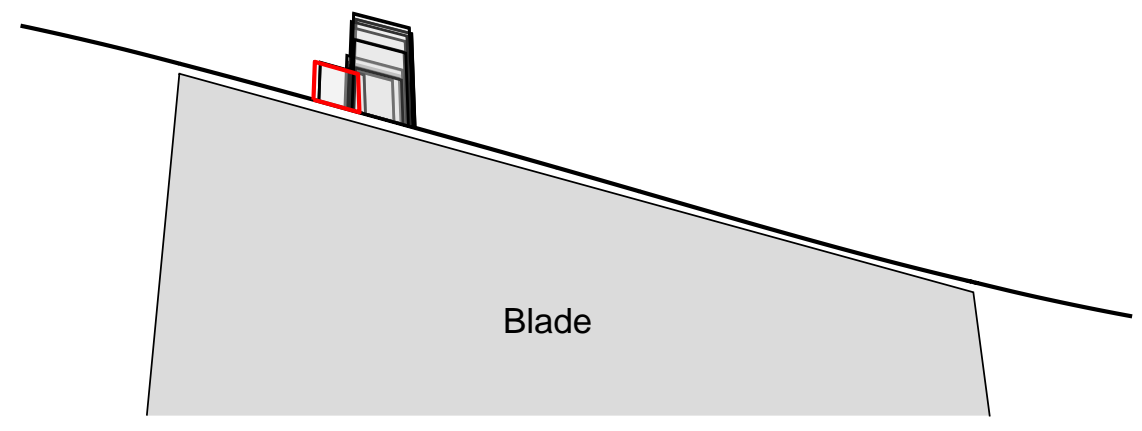

Figure 16 Pareto solution of casing groove designs
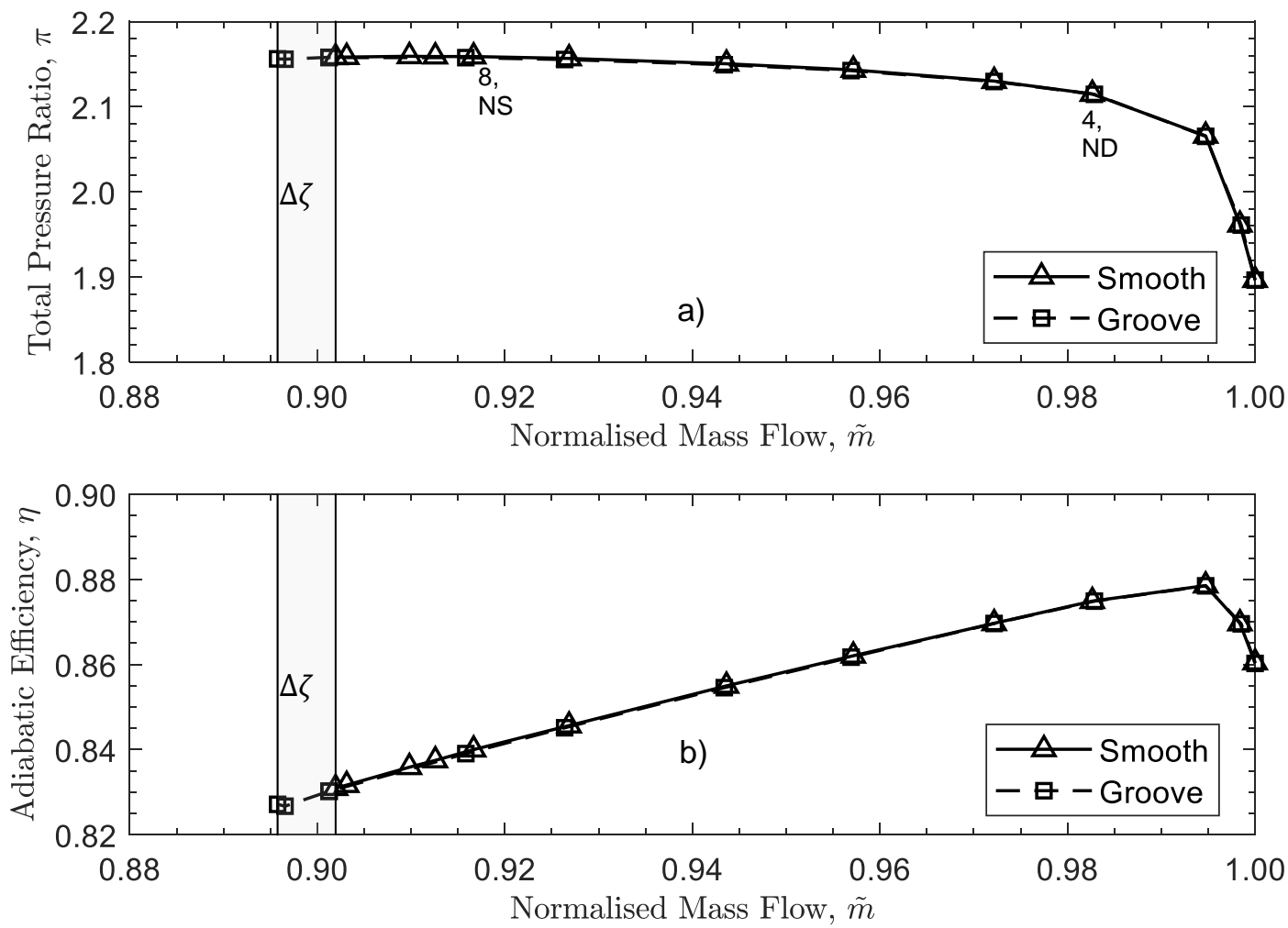

Figure 17 Optimum groove performance map a) Total pressure ratio and b) Adiabatic efficiency 


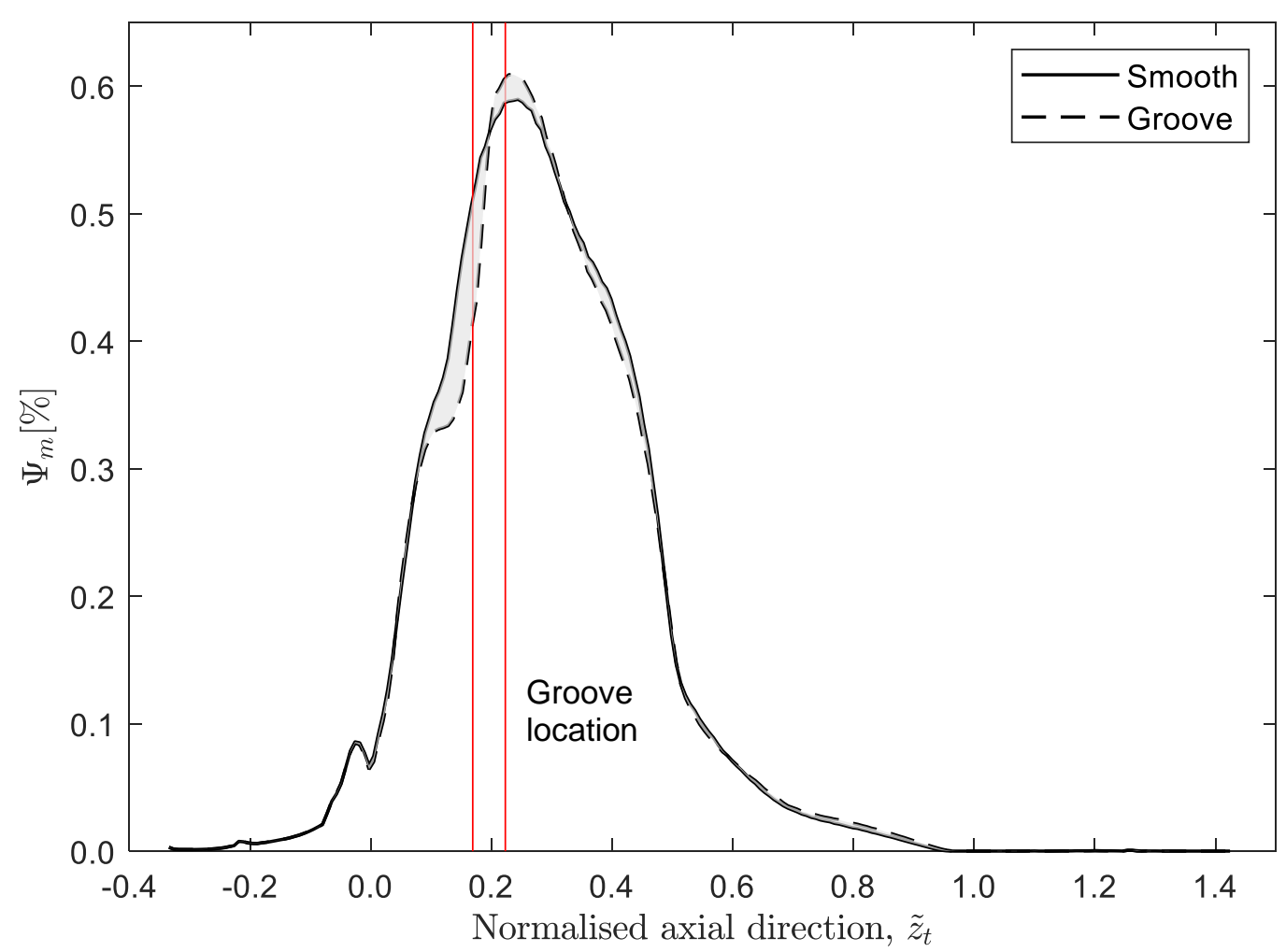

Figure 18 Comparison of $\Psi_{m}$ at point 8(NS) for smooth and grooved casing


Figure 19 Mach number contour for a) smooth and b) groove casing inside the tip gap 

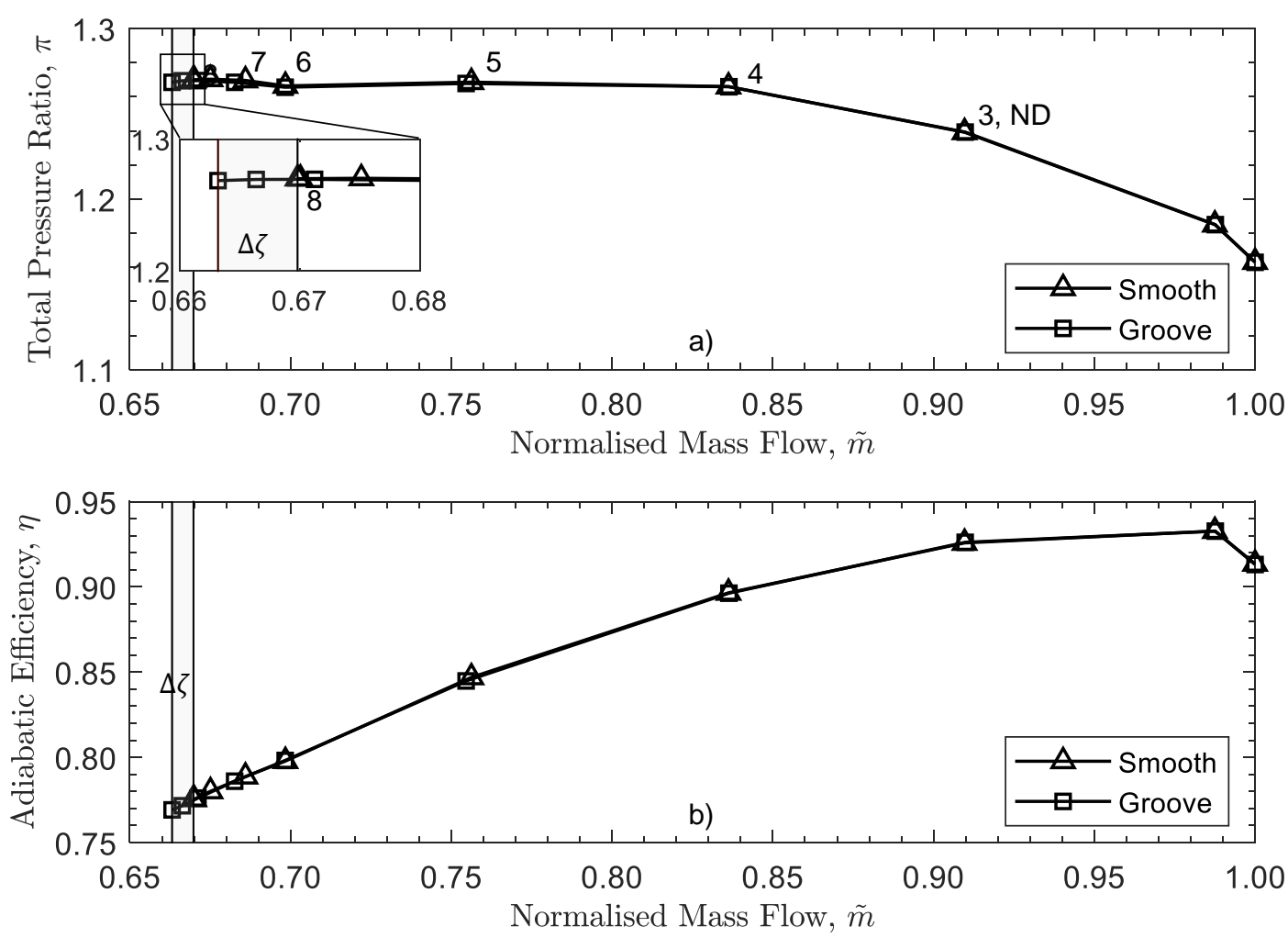

Figure 20 Performance characteristics at part-speed a) Total pressure ratio and b) Adiabatic efficiency

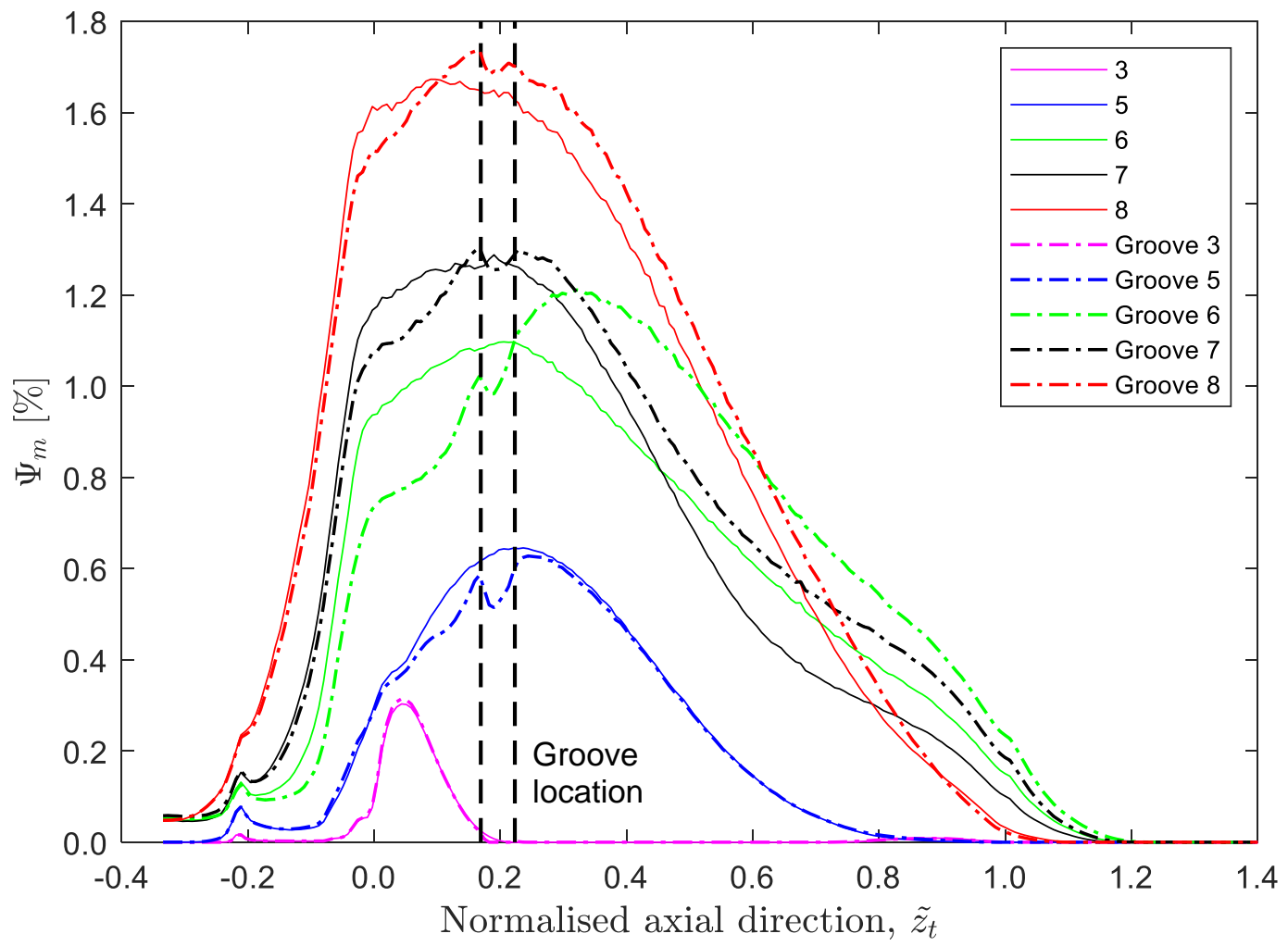

Figure 21 Comparison of $\Psi_{m}$ distribution for smooth and grooved casing 
Table 1 Design parameters of Rotor 37

\begin{tabular}{lcc}
\hline Blade count & 36 & \\
Total pressure ratio & 2.106 & \\
Rotational speed & 17188.7 & $\mathrm{rpm}$ \\
Tip speed & 454.14 & $\mathrm{~m} / \mathrm{s}$ \\
Tip clearance & 0.356 & $\mathrm{~mm}$ \\
Tip radius at LE & 253.7 & $\mathrm{~mm}$ \\
Hub/Tip ratio at LE & 0.7 & \\
\hline
\end{tabular}

Table 2 Casing groove design parameter limits

\begin{tabular}{ccccc}
\hline & $z^{\prime}$ & $w^{\prime}$ & $H$ & $\alpha\left(^{\circ}\right)$ \\
Lower & -0.2 & 0.05 & $0.1 w^{\prime}$ & 90 \\
Upper & 1.2 & 0.2 & $5 w^{\prime}$ & 115 \\
\hline
\end{tabular}

Table 3 Optimum casing groove parameters

\begin{tabular}{cccc}
\hline$z^{\prime}$ & $w^{\prime}$ & $H$ & $\alpha\left(^{\circ}\right)$ \\
0.169 & 0.054 & $0.89 \mathrm{w}^{\prime}$ & 92 \\
\hline
\end{tabular}

